This item was submitted to Loughborough's Research Repository by the author.

Items in Figshare are protected by copyright, with all rights reserved, unless otherwise indicated.

\title{
Kato's inequality for magnetic relativistic Schrödinger operators
}

\section{PLEASE CITE THE PUBLISHED VERSION}

http://www.ems-ph.org/journals/show_abstract.php?issn=0034-5318\&vol=53\&iss=1\&rank=3

\section{PUBLISHER}

(c) 2017 EMS Publishing House

\section{VERSION}

AM (Accepted Manuscript)

\section{PUBLISHER STATEMENT}

This work is made available according to the conditions of the Creative Commons Attribution-NonCommercialNoDerivatives 4.0 International (CC BY-NC-ND 4.0) licence. Full details of this licence are available at: https://creativecommons.org/licenses/by-nc-nd/4.0/

\section{LICENCE}

CC BY-NC-ND 4.0

\section{REPOSITORY RECORD}

Hiroshima, Fumio, Takashi Ichinose, and Jozsef Lorinczi. 2016. "Kato's Inequality for Magnetic Relativistic Schrödinger Operators”. Loughborough University. https://hdl.handle.net/2134/21216. 


\title{
Kato's Inequality for Magnetic Relativistic Schrödinger Operators
}

\author{
By \\ Fumio Hiroshima ${ }^{1)}$, Takashi Ichinose ${ }^{2)}$ and József Lőrinczi ${ }^{3)}$ \\ 1) Faculty of Mathematics, Kyushu University, 744 Motooka, Fukuoka, 819-0395, Japan; \\ hiroshima@math.kyushu-u.ac.jp \\ 2) Department of Mathematics, Kanazawa University, Kanazawa, 920-1192, Japan; \\ ichinose@kenroku.kanazawa-u.ac.jp \\ 3) Department of Mathematical Sciences, Loughborough University, Loughborough LE11 3TU, \\ United Kingdom; J.Lorinczi@lboro.ac.uk
}

\begin{abstract}
Kato's inequality is shown for the magnetic relativistic Schrödinger operator $H_{A, m}$ defined as the operator theoretical square root of the selfadjoint, magnetic nonrelativistic Schrödinger operator $(-i \nabla-A(x))^{2}+m^{2}$ with an $L_{\text {loc }}^{2}$ vector potential $A(x)$.
\end{abstract}

Mathematics Subject Classification (2010): 47A50; 81Q10; 47B25; 47N50; 47D06; $47 \mathrm{D} 08$.

Keywords: Kato's inequality; relativistic Schrödinger operator; magnetic relativistic Schrödinger operator.

Running Head: Kato's Inequality

Footnotes for the first page

Communicated by H. Okamoto. Received January 15, 2016. Revised June 13, 2016; July 11, 2016.

F. Hiroshima: Faculty of Mathematics, Kyushu University, 744 Motooka, Fukuoka, 819-0395, Japan; e-mail: hiroshima@math.kyushu-u.ac.jp

T. Ichinose: Department of Mathematics, Kanazawa University, Kanazawa, 920-1192, Japan; e-mail: ichinose@kenroku.kanazawa-u.ac.jp

J. Lőrinczi: Department of Mathematical Sciences, Loughborough University, Loughborough LE11 3TU, United Kingdom; e-mail: J.Lorinczi@lboro.ac.uk

$+++++++++++++++++++++++++++++++++++++$

The Corresponding Author:

Takashi ICHINOSE, E-mail: ichinose@kenroku.kanazawa-u.ac.jp,

Tel: +81-76-244-8631; Fax: +81-76-264-5738 


\section{Introduction}

Consider the magnetic relativistic Schrödinger operator

$$
H_{A, m}:=\sqrt{(-i \nabla-A(x))^{2}+m^{2}}
$$

in $d$-dimensional space $\mathbb{R}^{d}$ with vector potential $A(x):=\left(A_{1}(x), \ldots, A_{d}(x)\right)$ and rest mass $m \geq 0$, which may be thought of being a quantum Hamiltonian corresponding to the classical relativistic Hamiltonian symbol $\sqrt{(\xi-A(x))^{2}+m^{2}},(\xi, x) \in \mathbb{R}^{d} \times \mathbb{R}^{d}$. It is known that when $A(x)$ is an $\mathbb{R}^{d}$-valued function belonging to $\left[L_{\mathrm{loc}}^{2}\left(\mathbb{R}^{d}\right)\right]^{d} \equiv L_{\mathrm{loc}}^{2}\left(\mathbb{R}^{d} ; \mathbb{R}^{d}\right)$, it becomes a selfadjoint operator in $L^{2}\left(\mathbb{R}^{d}\right)$, which is essentially selfadjoint on $C_{0}^{\infty}\left(\mathbb{R}^{d}\right)$ so that $H_{A, m}$ has a domain containing $C_{0}^{\infty}\left(\mathbb{R}^{d}\right)$ as an operator core (e.g see [CFKiSi87, p.9]). We shall assume that $d \geq 2$, since in case $d=1$ any magnetic vector potential can be removed by a gauge tranformation. For $A=0$ we put $H_{0, m}=\sqrt{-\Delta+m^{2}}$, where $-\Delta$ is the minus-signed Laplacian $-\left(\frac{\partial^{2}}{\partial x_{1}^{2}}+\cdots+\frac{\partial^{2}}{\partial x_{d}^{2}}\right)$ as well as a nonnegative selfadjoint operator realized in $L^{2}\left(\mathbb{R}^{d}\right)$ having the Sobolev space $H^{2}\left(\mathbb{R}^{d}\right)$ as its domain.

The aim of this paper is to show Kato's inequality for this magnetic relativistic Schrödinger operator $H_{A, m}$ or $H_{A, m}-m$, when $A$ is an $\mathbb{R}^{d}$-valued $L_{\text {loc }}^{2}$ function in $\mathbb{R}^{d}$.

Theorem 1.1. (Kato's inequality). Let $m \geq 0$ and assume $A \in\left[L_{\mathrm{loc}}^{2}\left(\mathbb{R}^{d}\right)\right]^{d}$. If $u \in$ $L^{2}\left(\mathbb{R}^{d}\right)$ with $H_{A, m} u \in L_{\text {loc }}^{1}\left(\mathbb{R}^{d}\right)$, then the following distributional inequality holds:

$$
\operatorname{Re}\left[(\operatorname{sgn} u) H_{A, m} u\right] \geq H_{0, m}|u|,
$$

or

$$
\operatorname{Re}\left[(\operatorname{sgn} u)\left[H_{A, m}-m\right] u\right] \geq\left[H_{0, m}-m\right]|u| .
$$

Here sgn is a bounded function in $\mathbb{R}^{d}$ defined by

$$
(\operatorname{sgn} u)(x)= \begin{cases}\overline{u(x)} /|u(x)|, & \text { if } u(x) \neq 0, \\ 0, & \text { if } u(x)=0 .\end{cases}
$$

Note here that $H_{A, m} u$ with $u \in L^{2}\left(\mathbb{R}^{d}\right)$ makes sense as a distribution in $\mathbb{R}^{d}$ (for this, see Lemma 2.2 with $\alpha=1$ and a few lines after its proof). A characteristic feature in this situation is that $H_{A, m}$ is a nonlocal operator defined by the operator-theoretical square root of a nonnegative selfadjoint operator. It is not a differential operator, and neither an integral operator nor a pseudo-differential operator associated with a certain tractable symbol. The point which becomes crucial is in how to go without knowledge on regularity of the weak solution $u \in L^{2}\left(\mathbb{R}^{d}\right)$ of equation $H_{A, m} u=f$ for a given $f \in L_{\text {loc }}^{1}\left(\mathbb{R}^{d}\right)$. Thus the present inequality $(1.2) /(1.3)$ differs from an abstract form of Kato's inequality such as in [Si77] by being substatially sharp.

An immediate corollary is the following theorem, which has been known (e.g. [FLSei08], [HILo12]; cf. [I93]).

Theorem 1.2. (Diamagnetic inequality) Let $m \geq 0$ and assume that $A \in\left[L_{\mathrm{loc}}^{2}\left(\mathbb{R}^{d}\right)\right]^{d}$. Then it holds that for $f, g \in L^{2}\left(\mathbb{R}^{d}\right)$,

$$
\left|\left(f, e^{-t\left[H_{A, m}-m\right]} g\right)\right| \leq\left(|f|, e^{-t\left[H_{0, m}-m\right]}|g|\right) .
$$


Once Theorem 1.1 is established, we can apply it to show the following theorem on essential selfadjointness of the relativistic Schrödinger operator with both vector and scalar potentials $A(x)$ and $V(x)$ :

$$
H_{A, V, m}:=H_{A, m}+V .
$$

Theorem 1.3. Let $m \geq 0$, assume that $A \in\left[L_{\text {loc }}^{2}\left(\mathbb{R}^{d}\right)\right]^{d}$ and let $V \in L_{\text {loc }}^{2}\left(\mathbb{R}^{d}\right)$ with $V(x) \geq 0$ a.e. Then $H_{A, V, m}=H_{A, m}+V$ is essentially selfadjoint on $C_{0}^{\infty}\left(\mathbb{R}^{d}\right)$ and its unique selfadjoint extension is bounded below by $m$.

We shall show inequality (1.2)/(1.3), basically along the idea and method of Kato's original proof in [K72] for the magnetic nonrelativistic Schrödinger operator $\frac{1}{2}(-i \nabla-$ $A(x))^{2}$. As a matter of fact, we follow the method of proof modified for the existing form of Kato's inequality in [I89], [ITs92] for another magnetic relativistic Schrödinger operator which is defined as a Weyl pseudo-differential operator associated with the same relativistic classical symbol $\sqrt{(\xi-A(x))^{2}+m^{2}}$. However, this is not sufficient, and we need further modifications using operator theory, since pseudo-differential calculus does not seem useful. Starting from the assumption of the theorem that $u \in L^{2}$ and $H_{A, m} u \in L_{\mathrm{loc}}^{1}$, it appears to be impossible to show the regularity of $u$ that $\partial_{j} u \in L_{\text {loc }}^{1}, 1 \leq j \leq d$, and/or $H_{0, m} u \in L_{\text {loc }}^{1}$, which may be due to the fact that the operators $\partial_{j} \cdot\left(-\Delta+m^{2}\right)^{-1 / 2}, 1 \leq j \leq d$, are not bounded from $L^{1}$ to $L^{1}$, though they are bounded from $L^{1}$ to weak $L^{1}$-space. Therefore we make a detour by going via the case of the fractional power $\left(H_{A, m}\right)^{\alpha}$ with $\alpha<1$. Verifying that the assumption implies that $\left(H_{A, m}\right)^{\alpha} u \in L_{\mathrm{loc}}^{1}$ for $0<\alpha<1$, we show the asserted inequality first for the case $0<\alpha<1$, i.e. inequality $(1.2) /(1.3)$ with the pair $H_{A, m}, H_{0, m}$, replaced by the pair $\left(H_{A, m}\right)^{\alpha},\left(H_{0, m}\right)^{\alpha}$, respectively, and then for the case $\alpha=1$, appealing to the fact, to be shown, that $\left(H_{A, m}\right)^{\alpha} u$ converges to $H_{A, m} u$ in $L_{\text {loc }}^{1}$ as $\alpha \uparrow 1$. The proof is presented separately according to $m>0$ and $m=0$, in a self-contained manner.

A comment is in order on our starting assumption for $u$, namely, why the theorem is formulated with assumption that $u \in L^{2}$ and $H_{A, m} u \in L_{\text {loc }}^{1}$, but not that both $u$ and $H_{A, m} u$ are $L_{\mathrm{loc}}^{1}$. For this question, recall that the original form of Kato's inequality for nonrelativistic Schrödinger operators $\frac{1}{2}(-i \nabla-A(x))^{2}$ is formulated under the assumption that both $u$ and $\frac{1}{2}(-i \nabla-A(x))^{2} u$ are $L_{\mathrm{loc}}^{1}$. The answer is simply because of avoiding inessential complexity coming from the fact that $H_{A, m}$ is a nonlocal operator.

The relativistic Schrödinger operator $H_{0, m}=\sqrt{-\Delta+m^{2}}$ without vector potential was first considered in [W74] and [He77] for spectral problems. The magnetic relativistic Schrödinger operator $H_{A, m}$ like (1.1) is used to study problems related to "stability of matter" in relativistic quantum mechanics in [LSei10]. On the other hand, a problem of representing by path integral the relativistic Schrödinger semigroup with generator $H_{A, m}$ has been also studied. A result is a formula of Feynman-Kac-Itô type (cf. [Si79/05]), earlier in [DeRiSe91], [DeSe90] and also in [N00], which has been recently extensively studied in [HILo12], [HILo13] (cf. [LoHBe11]). The problem is connected with a Lévy process obtained by subordinating Brownian motion ([Sa99], [Ap04/09]). A weaker version of Kato's inequality as well as the diamagnetic inequality was given in our paper [HILo12], to which the present work adds further results.

In Section 2 some technical lemmas are given, which are used in the proof of theorems. They concern some basic inequalities in $L^{2}$ and $L^{p}$ connected with the semigroups and/or inverse (resolvent) for the magnetic nonrelativistic (but not relativistic) 
Schrödinger operator $(-i \nabla-A)^{2}+m^{2}$, which is the square of our magnetic relativistic Schrödinger operator $H_{A, m}$. For the sake of regularization of $H_{A, m}$, its fractional powers $\left(H_{A, m}\right)^{\alpha}$ with $0<\alpha<1$ are also considered through the semigroup of the magnetic nonrelativistic Schrödinger operator to estimate in local $L^{1}$-norm a kind of difference, being a distance in a particular sense, between $\left(H_{A, m}\right)^{\alpha}$ and $\left(H_{0, m}\right)^{\alpha}$, each applied to a function.

In Section 3 we prove the theorems. Section 4 is to make concluding remarks about how the issue is going with the other two magnetic relativistic Schrödinger operators associated with the same symbol. Appendix A provides for an explicit expression of the integral kernel (heat kernel) of the semigroup $e^{-t\left[\left(H_{0, m}\right)^{\alpha}-m^{\alpha}\right]}$ for the free fractional power $\left(H_{0, m}\right)^{\alpha}$ together with the density (function) of the associated Lévy measure $n^{m, \alpha}(d y)$. For basic facts on the magnetic relativistic Schrödinger operator, we refer, e.g., to [LLos01] and [BE11].

Finally, we note that we have defined the fractional powers of $H_{A, m}$ mainly through the magnetic nonrelativistic Schrödinger semigroup. However, an alternative way is to define them through the Dunford integral via the resolvent of the magnetic nonrelativistic Schrödinger operator.

\section{Technical Lemmas}

Throughout this paper, we denote by $(\cdot, \cdot)$ the Hilbert space inner product which is sesquilinear, i.e. conjugate-linear in the first argument and linear in the second (the physicist's convention), and by $\langle\cdot, \cdot\rangle$ the bilinear inner product which is linear in both the arguments.

Our main object of study is the operator $H_{A, m}:=\left[(-i \nabla-A)^{2}+m^{2}\right]^{\frac{1}{2}}$ in (1.1) with assumption that $A \in\left[L_{\text {loc }}^{2}\left(\mathbb{R}^{d}\right)\right]^{d}$, which is a selfadjoint operator in $L^{2}\left(\mathbb{R}^{d}\right)$ defined as the square root of the nonnegative selfadjoint (Schrödinger) operator $(-i \nabla-A)^{2}+m^{2}$ in $L^{2}\left(\mathbb{R}^{d}\right)$. For $m=0, H_{A, 0}=|-i \nabla-A|$. Among them, the following identity holds:

$$
\begin{aligned}
\left\|H_{A, m} u\right\|_{L^{2}}^{2} & =\left(u,\left(H_{A, m}\right)^{2} u\right)=\left(u,\left[(-i \nabla-A)^{2}+m^{2}\right] u\right) \\
& =\sum_{j=1}^{d}\left\|\left(-i \partial_{j}-A_{j}\right) u\right\|_{L^{2}}^{2}+m^{2}\|u\|_{L^{2}}^{2}=\left\|H_{A, 0} u\right\|_{L^{2}}^{2}+m^{2}\|u\|_{L^{2}}^{2},
\end{aligned}
$$

with $u \in C_{0}^{\infty}\left(\mathbb{R}^{d}\right)$ for all the five members and with $u$ in the domain of $H_{A, m}$ for the first, fourth and fifth members. The nonrelativistic Schrödinger op erator $(-i \nabla-A)^{2}+m^{2}$ concerned is the selfadjoint operator associated with this quadratic form (2.1), which has $C_{0}^{\infty}\left(\mathbb{R}^{d}\right)$ as a form core (e.g [CFKiSi87, 1.3, pp.8-9]). As a result, $H_{A, m}$ has $C_{0}^{\infty}\left(\mathbb{R}^{d}\right)$ as an operator core, in other words, $H_{A, m}$ is a nonnegative selfadjoint operator in $L^{2}\left(\mathbb{R}^{d}\right)$ having domain $D\left[H_{A, m}\right]:=\left\{u \in L^{2}\left(\mathbb{R}^{d}\right) ;\left(i \partial_{j}+A_{j}\right) u \in L^{2}\left(\mathbb{R}^{d}\right), \partial_{j}:=\right.$ $\left.\partial / \partial_{x_{j}}, 1 \leq j \leq d\right\}$, being essentially selfadjoint on $C_{0}^{\infty}\left(\mathbb{R}^{d}\right)$. Though $i \nabla+A \equiv$ $\left(i \partial_{1}+A_{1}, \ldots, i \partial_{d}+A_{d}\right)$ is a closed linear operator of $\left[L^{2}\left(\mathbb{R}^{d}\right)\right]^{d}$ into itself with domain $D[i \nabla+A]:=\left\{\left(u_{1}, \ldots, u_{d}\right) \in\left[L^{2}\left(\mathbb{R}^{d}\right)\right]^{d} ;\left(i \partial_{j}+A_{j}\right) u \in L^{2}\left(\mathbb{R}^{d}\right), \partial_{j}:=\partial / \partial_{x_{j}}, 1 \leq j \leq\right.$ $d\}$, we will also abuse notation to write the first term of the fourth member of (2.1) as $\|(-i \nabla-A) u\|_{L^{2}}^{2}$.

For the proof of Theorem 1.1, however, we need to consider $H_{A, m}$ also on $L^{p}$ spaces, and further the fractional powers $\left(H_{A, m}\right)^{\alpha}, 0<\alpha<1$ of $H_{A, m}$. The aim of this section concerns the issue such as some estimates connected with them. 
As for the constant $m$, unless otherwise stated, we assume in this section that $m>0$, and keep assuming it also in Section 3, until we come to consider the case including $m=0$ at the final stage of the proof of Theorem 1.1. Therefore, in case $m>0, H_{A, m}$ has bounded inverse $\left(H_{A, m}\right)^{-1}$, as well as $\left[(-i \nabla-A)^{2}+m^{2}\right]$ has bounded inverse $\left[(-i \nabla-A)^{2}+m^{2}\right]^{-1}$.

\subsection{Some inequalities related to magnetic nonrelativistic Schrödinger operators on $L^{p}$}

The operators $H_{A, m}$ may be considered not only in $L^{2}$ but also in $L^{p}, 1 \leq p<\infty$, in particular, for $p=1$. The square of $H_{A, m}$ becomes a magnetic nonrelativistic Schrödinger operator $(-i \nabla-A)^{2}+m^{2}$. Some basic inequalities are given which are related to the magnetic nonrelativistic Schrödinger semigroup $e^{-t\left(H_{A, m}\right)^{2}}$ and inverse (resolvent) $\left(\left(H_{A, m}\right)^{2}\right)^{-1}$ on $L^{p}$, though not with the magnetic relativistic Schrödinger semigroup $e^{-t H_{A, m}}$ and inverse (resolvent) $\left(H_{A, m}\right)^{-1}$. They will be useful throughout the paper.

In the beginning, let us repetitively confirm the notations to be used:

$$
\begin{aligned}
& \left(H_{A, m}\right)^{2}=(-i \nabla-A)^{2}+m^{2}, \quad\left(H_{A, 0}\right)^{2}=(-i \nabla-A)^{2}, \\
& \left(H_{0, m}\right)^{2}=-\Delta+m^{2}, \quad\left(H_{0,0}\right)^{2}=-\Delta .
\end{aligned}
$$

Lemma 2.1. Suppose $A \in\left[L_{\text {loc }}^{2}\left(\mathbb{R}^{d}\right)\right]^{d}$. Then the following inequalities hold.

(i) Let $1 \leq p \leq \infty$. For $m \geq 0$,

$$
\left\|e^{-t\left(H_{A, m}\right)^{2}}\right\|_{L^{p} \rightarrow L^{p}} \leq\left\|e^{-t\left(H_{0, m}\right)^{2}}\right\|_{L^{p} \rightarrow L^{p}} \equiv\left\|e^{-t\left(-\Delta+m^{2}\right)}\right\|_{L^{p} \rightarrow L^{p}} \leq e^{-m^{2} t} \leq 1, \quad t>0 .
$$

For $m>0$ and $\beta>0$,

$$
\left\|\left(\left(H_{A, m}\right)^{2}\right)^{-\beta}\right\|_{L^{p} \rightarrow L^{p}} \leq\left\|\left(\left(H_{0, m}\right)^{2}\right)^{-\beta}\right\|_{L^{p} \rightarrow L^{p}}, \quad t>0 .
$$

(ii) Let $1 \leq p<\infty$. The operators $e^{-t\left(H_{0,0}\right)^{2}}(-i \nabla)$ and $e^{-t\left(H_{0,0}\right)^{2}}(-\Delta)$ can be extended to be bounded operators on $\left[L^{p}\left(\mathbb{R}^{d}\right)\right]^{d}$ and $L^{p}\left(\mathbb{R}^{d}\right)$ :

$$
\left\|e^{-t\left(H_{0,0}\right)^{2}}(-i \nabla)\right\|_{\left[L^{p}\right]^{d} \rightarrow\left[L^{p}\right]^{d}} \leq C_{1 p} t^{-1 / 2}, \quad\left\|e^{-t\left(H_{0,0}\right)^{2}}(-\Delta)\right\|_{L^{p} \rightarrow L^{p}} \leq C_{2 p} t^{-1}, \quad t>0,
$$

with constants $C_{1 p}>0$ and $C_{2 p}$ independent of $t$.

(iii) Let $m \geq 0$. The operators $H_{A, m} e^{-\left(H_{A, m}\right)^{2}}$ and $\left(H_{A, m}\right)^{2} e^{-t\left(H_{A, m}\right)^{2}}$ can be extended to be bounded operators on $L^{2}\left(\mathbb{R}^{d}\right)$ :

$$
\left\|H_{A, m} e^{-t\left(H_{A, m}\right)^{2}}\right\|_{L^{2} \rightarrow L^{2}} \leq(2 e t)^{-1 / 2}, \quad\left\|\left(H_{A, m}\right)^{2} e^{-t\left(H_{A, m}\right)^{2}}\right\|_{L^{2} \rightarrow L^{2}} \leq(e t)^{-1}, \quad t>0 .
$$

(iv) The operators $e^{-t(-i \nabla-A)^{2}}(i \nabla+A)$ and $(i \nabla+A) e^{-t(-i \nabla-A)^{2}}$ can be extended to be bounded operators on $\left[L^{2}\left(\mathbb{R}^{d}\right)\right]^{d}$ :

$$
\begin{aligned}
& \left\|e^{-t(-i \nabla-A)^{2}}(i \nabla+A)\right\|_{\left[L^{2}\right]^{d} \rightarrow\left[L^{2}\right]^{d}} \leq\left(\frac{d}{2 e t}\right)^{\frac{1}{2}}, \\
& \left\|(i \nabla+A) e^{-t(-i \nabla-A)^{2}}\right\|_{\left[L^{2}\right]^{d} \rightarrow\left[L^{2}\right]^{d}} \leq\left(\frac{d}{2 e t}\right)^{\frac{1}{2}}, \quad t>0 .
\end{aligned}
$$


The assertion (ii) of Lemma 2.1 may be an $L^{p}$ version of (iii) or (iv) above, though only for a special case of the minus-signed Laplacian $-\Delta$ without vector potential $A(x)$.

Proof of Lemma 2.1. (i) This is due to the ingenious observation given for the magnetic nonrelativistic Schrödinger operator $(-i \nabla-A(x))^{2}$ with $A \in L_{\text {loc }}^{2}$ in [Si79, Theorem 2.3, p.40], [Si82, Sect. B13, p.490], since $\left(H_{A, m}\right)^{2}=(-i \nabla-A(x))^{2}+m^{2}$ is nothing but a magnetic (nonrelativistic) Schrödinger operator plus the constant $\mathrm{m}^{2}$. Following the arguments there we have, for $1 \leq p<\infty$ and for every $u \in C_{0}^{\infty}\left(\mathbb{R}^{d}\right)$,

$$
\left|e^{-t\left(H_{A, m}\right)^{2}} u\right| \leq e^{-t\left(H_{0, m}\right)^{2}}|u|=e^{-m^{2} t} e^{-t(-\Delta)}|u|, \quad \text { pointwise a.e., }
$$

so that $e^{-\left(H_{A, m}\right)^{2}} L^{p}\left(\mathbb{R}^{d}\right) \subseteq L^{\infty}\left(\mathbb{R}^{d}\right) \cap L^{p}\left(\mathbb{R}^{d}\right)$, in fact, for $u \in L^{p}\left(\mathbb{R}^{d}\right)$,

$$
\left\|e^{-t\left(H_{A, m}\right)^{2}} u\right\|_{L^{p}} \leq e^{-m^{2} t}\left\|e^{-t(-\Delta)}|u|\right\|_{L^{p}} \leq e^{-m^{2} t}\|u\|_{L^{p}} \leq\|u\|_{L^{p}}, \quad t \geq 0 .
$$

Thus we can consider $e^{-t\left(H_{A, m}\right)^{2}}$ also as a bounded linear operator mapping $L^{p}\left(\mathbb{R}^{d}\right)$ into itself. Moreover, it is seen it is a contraction semigroup. We may use the notations $\left(H_{A, m}\right)^{2}, H_{A, m}$ also to mean operators $\left(H_{A, m}\right)_{p}^{2},\left(H_{A, m}\right)_{p}$ in $L^{p}$ when there is no risk of confusion. Furthermore, for the crucial assertion (i), we refer to [Si82, Corollary B.13.3, p.491].

(ii) In fact, $e^{-t(-\Delta)}$ becomes a holomorphic semigroup on $L^{p}\left(\mathbb{R}^{d}\right), 1 \leq p<\infty$, for $\operatorname{Re} t>0$. Then for any $f \in L^{p}\left(\mathbb{R}^{d}\right), v(t):=e^{-t(-\Delta)} f$ gives a unique solution of the heat equation $\frac{\partial}{\partial t} v(t)=\Delta v(t)$ (see e.g. [K76, IX.§1.8, p.495] and [K76, IX.§1.6, Remark $1.22, \mathrm{p} .492])$. This implies that $e^{-t(-\Delta)}$ has range in the domain $D[(-\Delta)]$ of $(-\Delta)$, equivalently, that $t e^{-t(-\Delta)}(-\Delta)$ is uniformly bounded from $L^{p}\left(\mathbb{R}^{d}\right)$ into itself for every real $t>0$, and so is $t^{1 / 2} e^{-t(-\Delta)}\left(-i \partial_{j}\right)$ for each $j=1,2, \ldots, d$.

(iii) For functions in in $L^{2}$, the assertion are evident by the spectral theorem, because $\left(H_{A, m}\right)^{2}$ and $H_{A, m}$ are nonnegative selfadjoint operators in the Hilbert space $L^{2}\left(\mathbb{R}^{d}\right)$. Indeed, it is easy to see that for $u \in C_{0}^{\infty}\left(\mathbb{R}^{d}\right)$,

$$
\begin{aligned}
& \left\|e^{-t\left(H_{A, m}\right)^{2}} H_{A, m} u\right\|_{L^{2}}^{2}=\left(u,\left(H_{A, m}\right)^{2} e^{-2 t\left(H_{A, m}\right)^{2}} u\right) \leq \sup _{\lambda \geq 0} \lambda e^{-2 t \lambda}\|u\|_{L^{2}}^{2}=(2 e t)^{-1}\|u\|_{L^{2}}^{2}, \\
& \left\|e^{-t\left(H_{A, m}\right)^{2}}\left(H_{A, m}\right)^{2} u\right\|_{L^{2}}^{2}=\left(u,\left(H_{A, m}\right)^{4} e^{-2 t\left(H_{A, m}\right)^{2}} u\right) \leq \sup _{\lambda \geq 0} \lambda^{2} e^{-2 t \lambda}\|u\|_{L^{2}}^{2}=(e t)^{-2}\|u\|_{L^{2}}^{2} .
\end{aligned}
$$

This shows (iii).

(iv) (proof) These inequalities follow from (ii). Indeed, for the first one, since

$$
\left\|e^{-t(-i \nabla-A)^{2}}(i \nabla+A) \varphi\right\|_{L^{2}}^{2}:=\sum_{j=1}^{d}\left\|e^{-t \sum_{k=1}^{d}\left(-i \partial_{k}-A_{k}\right)^{2}}\left(i \partial_{j}+A_{j}\right) \varphi_{j}\right\|_{L^{2}}^{2}
$$

for $\varphi=\left(\varphi_{1}, \ldots, \varphi_{d}\right) \in\left[C_{0}^{\infty}\left(\mathbb{R}^{d}\right)\right]^{d}$, we have only to show that for each $j$

$$
\left\|e^{-t(-i \nabla-A)^{2}}\left(i \partial_{j}+A_{j}\right) \varphi_{j}\right\|_{L^{2}}^{2} \leq(2 e t)^{-1}\left\|\varphi_{j}\right\|_{L^{2}}^{2} .
$$

This is seen as follows: For $m>0$, we have by (ii)

$$
\begin{aligned}
& \left\|e^{-t(-i \nabla-A)^{2}}\left(i \partial_{j}+A_{j}\right) \varphi_{j}\right\|_{L^{2}}^{2} \\
& =e^{2 m^{2}}\left\|\left[e^{-t H_{A, m}^{2}} H_{A, m}\right]\left[H_{A, m}^{-1}\left(\left(i \partial_{j}+A_{j}\right)^{2}+m^{2}\right)^{1 / 2}\right]\left[\left(\left(i \partial_{j}+A_{j}\right)^{2}+m^{2}\right)^{-1 / 2}\left(i \partial_{j}+A_{j}\right)\right] \varphi_{j}\right\|_{L^{2}}^{2} \\
& \leq e^{2 m^{2}}(2 e t)^{-1}\left\|\left[H_{A, m}^{-1}\left(\left(i \partial_{j}+A_{j}\right)^{2}+m^{2}\right)^{1 / 2}\right]\left[\left(\left(i \partial_{j}+A_{j}\right)^{2}+m^{2}\right)^{-1 / 2}\left(i \partial_{j}+A_{j}\right)\right] \varphi_{j}\right\|_{L^{2}}^{2} \\
& \leq e^{2 m^{2}}(2 e t)^{-1}\left\|\varphi_{j}\right\|_{L^{2}}^{2} .
\end{aligned}
$$


Letting $m \downarrow 0$, we have the result.

The second one is shown similarly. This shows (iv), ending the proof of Lemma 2.1 .

Remark. Nontriviality of the assertion (ii) of this lemma lies in that $i \nabla+A$ does not commute with the operator $(i \nabla+A(x))^{2}=\sum_{j=1}^{d}\left(i \partial_{j}+A_{j}(x)\right)^{2}$ or $\left(H_{A, m}\right)^{2}$

\subsection{Estimate of a kind of difference between $\left(H_{A, m}\right)^{\alpha}$ and $\left(H_{0, m}\right)^{\alpha}$ in local $L^{1}$-norm}

In this subsection, we consider the operators given by the fractional powers $\left(H_{A, m}\right)^{\alpha}:=$ $\left[(-i \nabla-A)^{2}+m^{2}\right]^{\alpha / 2}, 0<\alpha \leq 1$, and provide several lemmas to estimate in local $L^{1}$-norm a kind of difference between $\left(H_{A, m}\right)^{\alpha}$ and $\left(H_{0, m}\right)^{\alpha}$, each applied to a function $u$. They are needed to prove Theorem 1.1. Of course, the case for $\alpha=1$ turns out to be our operator itself: $\left(H_{A, m}\right)^{1} \equiv H_{A, m}=\left[(-i \nabla-A)^{2}+m^{2}\right]^{\frac{1}{2}}$.

Given a positive self-adjoint operator $S$ in a Hilbert space $L^{2}\left(\mathbb{R}^{d}\right)$ with domain $D[S]$, we adopt the following definition of its fractional powers $S^{\alpha}$ to be suggested from the identity for the gamma function $\Gamma(\beta), s^{-\beta}=\frac{1}{\Gamma(\beta)} \int_{0}^{\infty} t^{\beta-1} e^{-s t} d t$ with $t>0$ and $0<\beta \leq 1$ : for $0 \leq \alpha<1$,

$$
S^{\alpha} u=S^{-(1-\alpha)} \cdot S u=\frac{1}{\Gamma(1-\alpha)} \int_{0}^{\infty} t^{-\alpha} e^{-t S} S u d t, \quad u \in D[S] .
$$

We shall use these formulas, taking for $S$ the nonrelativistic Schrödinger operator $\left[(-i \nabla-A)^{2}+m^{2}\right]=\left(H_{A, m}\right)^{2}$ and/or $\left[-\Delta+m^{2}\right]=\left(H_{0, m}\right)^{2}$, but not the relativistic Schrödinger operator $H_{A, m}$ and/or $H_{0, m}$. Thus for $f \in L^{2}\left(\mathbb{R}^{d}\right)$,

$$
\begin{aligned}
\left(H_{A, m}\right)^{-\beta} f & =\left[(-i \nabla-A)^{2}+m^{2}\right]^{-\frac{\beta}{2}} f \\
& =\frac{1}{\Gamma\left(\frac{\beta}{2}\right)} \int_{0}^{\infty} t^{\frac{\beta}{2}-1} e^{-t\left[(-i \nabla-A)^{2}+m^{2}\right]} f d t \quad(0<\beta \leq 2),
\end{aligned}
$$

and similarly for $\left(H_{0, m}\right)^{-\beta} \equiv\left[-\Delta+m^{2}\right]^{-\beta / 2}$ in case $A=0$. Therefore, for $u \in C_{0}^{\infty}\left(\mathbb{R}^{d}\right)$, we have

$$
\begin{aligned}
\left(H_{A, m}\right)^{\alpha} u & =\left[(-i \nabla-A)^{2}+m^{2}\right]^{\alpha / 2} u \\
& =\frac{1}{\Gamma\left(\frac{2-\alpha}{2}\right)} \int_{0}^{\infty} t^{\frac{2-\alpha}{2}-1} e^{-t\left[(-i \nabla-A)^{2}+m^{2}\right]}\left[(-i \nabla-A)^{2}+m^{2}\right] u d t \\
& =\frac{1}{\Gamma\left(\frac{2-\alpha}{2}\right)} \int_{0}^{\infty} t^{-\frac{\alpha}{2}} e^{-t\left(H_{A, m}\right)^{2}}\left(H_{A, m}\right)^{2} u d t, \quad(0 \leq \alpha<2),
\end{aligned}
$$

for $u$ in the domain of $\left(H_{A, m}\right)^{2}$, and similarly for $\left(H_{0, m}\right)^{\alpha} \equiv\left[-\Delta+m^{2}\right]^{\alpha / 2}$ in case $A=0$. Here note that $H_{A, m} / H_{0, m}$, as well as $S=(-i \nabla-A)^{2}+m^{2} /\left(-\Delta+m^{2}\right)$, has bounded inverse, since we are assuming in this section that $m>0$. It may be instructive to recognize that for $0<\alpha<1$ the last integral of (2.3) exists not only for $u \in D\left[\left(H_{A, m}\right)^{2}\right]$ but also for $u \in D\left[H_{A, m}\right]$, because by Lemma 2.1(iii)

$$
t^{-\frac{\alpha}{2}}\left\|e^{-t\left(H_{A, m}\right)^{2}}\left(H_{A, m}\right)^{2} u\right\|_{L^{2}} \leq t^{-\frac{\alpha}{2}}\left\|e^{-t\left(H_{A, m}\right)^{2}} H_{A, m}\right\|\left\|H_{A, m} u\right\|_{L^{2}}=O\left(t^{-\frac{(1+\alpha)}{2}}\right) .
$$


Lemma 2.2. Let $0<\alpha \leq 1$. Assume that $A \in\left[L_{\mathrm{loc}}^{2}\left(\mathbb{R}^{d}\right)\right]^{d}$. Then if $\varphi \in C_{0}^{\infty}\left(\mathbb{R}^{d}\right)$, then $\left(H_{A, m}\right)^{\alpha} \varphi \in L^{2}\left(\mathbb{R}^{d}\right)$. In fact, it holds for every compact subset $K$ in $\mathbb{R}^{d}$ that

$$
\left\|\left(H_{A, m}\right)^{\alpha} \varphi\right\|_{L^{2}} \leq\left[|K|^{\frac{1}{2}}\left[\left(\left(m^{2}+1\right)^{\frac{1}{2}}+1\right)+\||A|\|_{L^{2}(K)}\right]\left[\|\nabla \varphi\|_{L^{\infty}(K)}+\|\varphi\|_{L^{\infty}(K)}\right],\right.
$$

for all $\varphi \in C_{0}^{\infty}\left(\mathbb{R}^{d}\right)$ with supp $\varphi \subseteq K$, where $|K|$ denotes the volume (Lebesgue measure) of $K$.

Proof. Let $\varphi \in C_{0}^{\infty}\left(\mathbb{R}^{d}\right)$ with $\operatorname{supp} \varphi \subseteq K$. Then for $0<\alpha \leq 1$, we have

$$
\begin{aligned}
\left\|\left(H_{A, m}\right)^{\alpha} \varphi\right\|_{L^{2}}^{2} & =\left(\varphi,\left(H_{A, m}\right)^{2 \alpha} \varphi\right)=\left(\varphi,\left[(-i \nabla-A)^{2}+m^{2}\right]^{\alpha} \varphi\right) \\
& \leq\left(\varphi,\left[(-i \nabla-A)^{2}+m^{2}+1\right]^{\alpha} \varphi\right) \\
& \leq\left(\varphi,\left[(-i \nabla-A)^{2}+m^{2}+1\right] \varphi\right) \\
& =\|(-i \nabla-A) \varphi\|_{L^{2}}^{2}+\left(m^{2}+1\right)\|\varphi\|_{L^{2}}^{2}=\left\|H_{A,\left(m^{2}+1\right)^{\frac{1}{2}}} \varphi\right\|_{L^{2}}^{2} .
\end{aligned}
$$

Here for the first term of the last second member recall our informal notation mentioned after (2.1). Hence

$$
\begin{aligned}
& \left\|\left(H_{A, m}\right)^{\alpha} \varphi\right\|_{L^{2}} \\
& \leq\|\nabla \varphi\|_{L^{2}}+\|A \varphi\|_{L^{2}}+\left(m^{2}+1\right)^{\frac{1}{2}}\|\varphi\|_{L^{2}} \\
& \leq|K|^{1 / 2}\|\nabla \varphi\|_{L^{\infty}(K)}+\||A|\|_{L^{2}(K)}\|\varphi\|_{L^{\infty}(K)}+\left(m^{2}+1\right)^{\frac{1}{2}}|K|^{1 / 2}\|\varphi\|_{L^{\infty}(K)}<\infty,
\end{aligned}
$$

which is finite by assumption on $A$ and $\varphi$. This shows the desired assertion. by

By this lemma, for $0<\alpha \leq 1$ we can define a distribution $\left(H_{A, m}\right)^{\alpha} u$ for $u \in L^{2}\left(\mathbb{R}^{d}\right)$

$$
\left\langle\left(H_{A, m}\right)^{\alpha} u, \phi\right\rangle=\left\langle u,\left(H_{-A, m}\right)^{\alpha} \phi\right\rangle=\int\left(u\left(H_{-A, m}\right)^{\alpha} \phi\right)(x) d x
$$

or

$$
\left(\left(H_{A, m}\right)^{\alpha} u, \phi\right)=\left(u,\left(H_{A, m}\right)^{\alpha} \phi\right)=\int\left(\bar{u}\left(H_{A, m}\right)^{\alpha} \phi\right)(x) d x,
$$

for $\phi \in C_{0}^{\infty}\left(\mathbb{R}^{d}\right)$, because, for every compact set $K$ in $\mathbb{R}^{d}$, we have

$$
\begin{aligned}
\left|\left(\left(H_{A, m}\right)^{\alpha} u, \phi\right)\right|= & \left|\left(u,\left(H_{A, m}\right)^{\alpha} \phi\right)\right| \leq\|u\|_{L^{2}}\left\|\left(H_{A, m}\right)^{\alpha} \phi\right\|_{L^{2}} \\
\leq\|u\|_{L^{2}}\left[\left(|K|^{\frac{1}{2}}\left(\left(m^{2}+1\right)^{\frac{1}{2}}+1\right)\right)+\||A|\|_{L^{2}(K)}\right] & \times\left[\|\nabla \phi\|_{L^{\infty}(K)}+\|\phi\|_{L^{\infty}(K)}\right],
\end{aligned}
$$

for all $\phi \in C_{0}^{\infty}\left(\mathbb{R}^{d}\right)$ with $\operatorname{supp} \phi \subseteq K$. This says that $\left(H_{A, m}\right)^{\alpha} u$ is a continuous linear functional on $C_{0}^{\infty}\left(\mathbb{R}^{d}\right)$, and so a distribution on $\mathbb{R}^{d}$.

Next, we study some properties of $\left(H_{A, m}\right)^{\alpha}$ in the case $A \equiv 0$, namely, $\left(H_{0, m}\right)^{\alpha} \equiv$ $\left(-\Delta+m^{2}\right)^{\alpha / 2}, 0<\alpha \leq 1$. This is the $\frac{\alpha}{2}$-power of the nonnegative selfadjoint operator $H_{0, m} \equiv-\Delta+m^{2}$ on $L^{2}\left(\mathbb{R}^{d}\right)$ or also a pseudo-differential operator defined through Fourier transform having the symbol $\left(|\xi|^{2}+m^{2}\right)^{\alpha / 2}$. The function $\xi \mapsto$ $\left(|\xi|^{2}+m^{2}\right)^{\alpha / 2}-m^{\alpha}$ is conditionally negative definite in $\mathbb{R}^{d}$ (e.g. [ReSi78, Appendix 2 to XIII.12, pp. 212-222]; [IkW81/89, p.65]), so that, for each fixed $t>0$, the function $e^{-t\left[\left(|\xi|^{2}+m^{2}\right)^{\alpha / 2}-m^{\alpha}\right]}$ is positive definite. We note that this is a specific case of a 
Bernstein function, providing the kinetic term of more general non-local Schrödinger operators which we have studied in HILo12.

As a result, its Fourier transform is a nonnegative function for each $t>0$, which is nothing but the integral kernel $k_{0}^{m, \alpha}(t, x)$ of the semi-group $e^{-t\left[\left(H_{0, m}\right)^{\alpha}-m^{\alpha}\right]}$ satisfying $\int_{\mathbb{R}^{d}} k_{0}^{m, \alpha}(t, x) d x=1$. We see further the operator $\left(H_{0, m}\right)^{\alpha} u$, say with $u \in C_{0}^{\infty}\left(\mathbb{R}^{d}\right)$, have an integral operator representation:

$$
\begin{aligned}
\left(\left(H_{0, m}\right)^{\alpha} u\right)(x) & \equiv\left(\left[-\Delta+m^{2}\right]^{\frac{\alpha}{2}} u\right)(x) \equiv\left(\mathcal{F}^{-1}\left(|\xi|^{2}+m^{2}\right)^{\frac{\alpha}{2}} \mathcal{F} u\right)(x) \\
& =m^{\alpha} u(x)-\int_{|y|>0}\left[u(x+y)-u(x)-I_{\{|y|<1\}} y \cdot \nabla_{x} u(x)\right] n^{m, \alpha}(d y),
\end{aligned}
$$

where $n^{m, \alpha}(d y)$ is a $\sigma$-finite measure on $\mathbb{R}^{d} \backslash\{0\}$ depending on $m \geq 0$ and $0<\alpha \leq 1$, called Lévy measure, which satisfies $\int_{|y|>0} \frac{|y|^{2}}{1+|y|^{2}} n^{m, \alpha}(d y)<\infty$. The Lévy measure is known [IkW62, Example.1, p.81] to be given from $k_{0}^{m, \alpha}(t, x)$ through

$$
\frac{1}{t} k_{0}^{m, \alpha}(t, d y) \quad \rightarrow \quad n^{m, \alpha}(d y), \quad t \downarrow 0 .
$$

In our case, it has density: $n^{m, \alpha}(d y)=n^{m, \alpha}(y) d y$.

For the expressions for the integral kernel $k_{0}^{m, \alpha}(t, x)$ of $e^{-t\left[\left(H_{0, m}\right)^{\alpha}-m^{\alpha}\right]}$ and the density (function) $n^{m, \alpha}(y)$, see Appendix A, (A.2). For $\alpha=1$, they are explicitly given (e.g. [I89, (2.4ab), (2.2ab), pp.268-269], [LLos01, 7.11 (11)]) as

$$
\begin{aligned}
& k_{0}^{m, 1}(t, x)= \begin{cases}2\left(\frac{m}{2 \pi}\right)(d+1) / 2 \frac{t e^{m t} K_{(d+1) / 2}\left(m\left(x^{2}+t^{2}\right)^{1 / 2}\right)}{\left(x^{2}+t^{2}\right)^{(d+1) / 4}}, & m>0, \\
\frac{\Gamma\left(\frac{d+1}{2}\right)}{\pi^{(d+1) / 2}} \frac{t}{\left(x^{2}+t^{2}\right)^{(d+1) / 2}}, & m=0 ;\end{cases} \\
& n^{m, 1}(y)= \begin{cases}2\left(\frac{m}{2 \pi}\right)(d+1) / 2 \frac{K_{(d+1) / 2}(m|y|)}{|y|^{(d+1) / 2}}, & m>0, \\
\frac{\Gamma\left(\frac{d+1}{2}\right)}{\pi^{(d+1) / 2}} \frac{1}{|y|^{d+1}}, & m=0,\end{cases}
\end{aligned}
$$

where $K_{\nu}(\tau)$ is the modified Bessel function of the third kind of order $\nu$, which satisfies $0<K_{\nu}(\tau) \leq C \max \left\{\tau^{-\nu}, \tau^{-\frac{1}{2}}\right\} e^{-\tau}, \tau>0$ with a constant $C>0$ when $\nu \geq \frac{1}{2}$.

For our later use, let us calculate the commutator $\left[\left(H_{A, m}\right)^{2}, \psi\right]$ with $\psi \in C_{0}^{\infty}\left(\mathbb{R}^{d}\right)$. Here for two operators $U$ and $V$, their commutator is denoted by $[U, V]:=U V-V U$. We have

$$
\begin{aligned}
{\left[\left(H_{A, m}\right)^{2}, \psi\right] } & =(-i \nabla-A)^{2} \psi-\psi(-i \nabla-A)^{2} \\
& =(i \nabla+A)(i \nabla \psi)+(i \nabla \psi)(i \nabla+A) \\
& =[(\Delta \psi)+2(i \nabla+A)(i \nabla \psi)] \text { or }=[(-\Delta \psi)+2(i \nabla \psi)(i \nabla+A)],
\end{aligned}
$$

as quadratic forms, i.e. for suitable functions $u, v$ on $\mathbb{R}^{d}$,

$$
\begin{aligned}
\left(u,\left[\left(H_{A, m}\right)^{2}, \psi\right] v\right) & =((i \nabla \psi)(i \nabla+A) u, v)+(u,(i \nabla \psi)(i \nabla+A) v) \\
& =(u,(\Delta \psi) v)+2(u,(i \nabla+A)(i \nabla \psi) v) \\
\text { or } & =(u,(-\Delta \psi) v)+2(u,(i \nabla \psi)(i \nabla+A) v) .
\end{aligned}
$$


Here note that $[i \nabla+A, \psi] v=(i \nabla \psi) v$ as well as $[i \nabla+A,(i \nabla \psi)] v=(-\Delta \psi) v$. In fact, it holds more generally with two $\mathbb{R}^{d}$-valued functions $A$ and $B$ that for a function $v$ in $\mathbb{R}^{d}$

$$
\begin{aligned}
& {\left[\left(H_{A, m}\right)^{2} \psi-\psi\left(H_{B, m}\right)^{2}\right] v} \\
& =(i \nabla+A)((i \nabla \psi)+\psi A) v+((i \nabla \psi)-\psi B)(i \nabla+B) v+\psi A(i \nabla v)-i \nabla(\psi B v) .
\end{aligned}
$$

Indeed, the left-hand side of (2.11) can be seen to be equal to

$$
\begin{aligned}
& {\left[(-i \nabla-A)^{2} \psi-\psi(-i \nabla-B)^{2}\right] v} \\
& \begin{array}{c}
=[(i \nabla+A)(i \nabla+A) \psi-\psi(i \nabla+B)(i \nabla+B)] v \\
=(i \nabla+A)[((i \nabla \psi)+\psi A) v+(i \nabla(\psi v)-(i \nabla \psi) v)] \\
\quad+[((i \nabla \psi)-\psi B)-(\psi(i \nabla)+(i \nabla \psi))](i \nabla+B) v \\
=(i \nabla+A)((i \nabla \psi)+\psi A) v+(i \nabla+A)(\psi i \nabla v) \\
\quad+((i \nabla \psi)-\psi B)(i \nabla+B) v-i \nabla(\psi(i \nabla+B) v) \\
=(i \nabla+A)((i \nabla \psi)+\psi A) v+((i \nabla \psi)-\psi B)(i \nabla+B) v+\psi A(i \nabla v)-i \nabla(\psi B v) .
\end{array}
\end{aligned}
$$

This shows (2.11). Taking $B=A$ in (2.11) yields the third member of (2.10), which implies the fourth and fifth members.

For the next lemma, we briefly mention the weak $L^{1}$-space $L_{w}^{1}(X)$, given a measurable subset $X$ of $\mathbb{R}^{d}$. It is by definition the linear space of all measurable function $f$ on $X$ such that

$$
\|f\|_{L_{w}^{1}}:=\sup _{a>0} a|\{x \in X ;|f(x)|>a\}|
$$

is finite, where $|Y|$ denotes the volume (Lebesgue measure) of the measurable set $Y \subseteq \mathbb{R}^{d} . L_{w}^{1}(X)$ is not a Banach space, because $\|f\|_{L_{w}^{1}}$ is not a norm but a quasi-norm, as it does not satisfy the triangle inequality. However, it holds that $\|f+g\|_{L_{w}^{1}} \leq$ $2\left(\|f\|_{L_{w}^{1}}+\|g\|_{L_{w}^{1}}\right)$. It is shown that $L_{w}^{1}(X)$ is a quasi-normed complete linear space (see e.g. [G10, Def.1.1.5, pp.5-6]). We have $\|f\|_{L_{w}^{1}} \leq\|f\|_{L^{1}}$, so that $L^{1}(X) \subseteq L_{w}^{1}(X)$. If $f_{n} \rightarrow f$ in $L_{w}^{1}$, then the $\left\{f_{n}\right\}$ converges to $f$ in measure (e.g. [G10, Prop.1.1.9, p.7]). We say " $f$ is locally in $L_{w}^{1}$ ", if for every compact set $K$ in $\mathbb{R}^{d}, f$ belongs to $L_{w}^{1}(K)$. In some literatures $L_{w}^{1}(X)$ may be denoted also by $L^{1, \infty}(X)$ (Lorentz space).

Lemma 2.3. Let $0<\alpha \leq 1$. Let $\psi \in C_{0}^{\infty}\left(\mathbb{R}^{d}\right)$. Then for the commutator $\left[\left(H_{0, m}\right)^{\alpha}, \psi\right]$, it holds, with a constant $C_{\alpha}$ dependent on $\psi$ and $\alpha$ but independent of $m \geq 0$, that (i) for $1<p<\infty$,

$$
\left\|\left[\left(H_{0, m}\right)^{\alpha}, \psi\right] u\right\|_{L^{p}}=\left\|\left(H_{0, m}\right)^{\alpha}(\psi u)-\psi\left(H_{0, m}\right)^{\alpha} u\right\|_{L^{p}} \leq C_{\alpha}\|u\|_{L^{p}},
$$

for all $u \in L^{p}\left(\mathbb{R}^{d}\right)$. Therefore if both $u$ and $\left(H_{0, m}\right)^{\alpha}(\psi u)$ are in $L^{p}$, then $\psi\left(H_{0, m}\right)^{\alpha} u$ is in $L^{p}$, and

$$
\left\|\psi\left(H_{0, m}\right)^{\alpha} u\right\|_{L^{p}} \leq C_{\alpha}\|u\|_{L^{p}}+\left\|\left(H_{0, m}\right)^{\alpha}(\psi u)\right\|_{L^{p}}
$$

(ii) for $p=1$,

$$
\left\|\left[\left(H_{0, m}\right)^{\alpha}, \psi\right] u\right\|_{L_{w}^{1}}=\left\|\left(H_{0, m}\right)^{\alpha}(\psi u)-\psi\left(H_{0, m}\right)^{\alpha} u\right\|_{L_{w}^{1}} \leq C_{\alpha}\|u\|_{L^{1}},
$$

for all $u \in L^{1}\left(\mathbb{R}^{d}\right)$. 
Remark. Inequality (2.13) does not hold for $p=1$, and instead we have (2.14) with the $L^{1}$-norm on the left-hand side replaced by the $L_{w}^{1}$-quasi-norm. This is dependent on the Calderón-Zygmund theorem (For this see Proposition 2.4 below).

Proof of Lemma 2.3. (i) As the second-half assertion follows from the first, i.e. inequality (2.13), we have only to show (2.13), and even only for $u \in C_{0}^{\infty}\left(\mathbb{R}^{d}\right)$, since $C_{0}^{\infty}\left(\mathbb{R}^{d}\right)$ is dense in $L^{2}\left(\mathbb{R}^{d}\right)$. The proof for the case $\alpha=1$ was given in [ITs92, p.274, Lemma 2.3] by using the integral operator representation (2.6) of $H_{0, m}=\sqrt{-\Delta+m^{2}}$. The proof for the case $0<\alpha<1$ is similar. So we only give an outline.

Use (2.6) to rewrite $\left[\left(H_{0, m}\right)^{\alpha}, \psi\right]$ as

$$
\begin{aligned}
\left(\left[\left(H_{0, m}\right)^{\alpha}, \psi\right] u\right)(x)= & -\int_{|y|>0}\left[\psi(x+y)-\psi(x)-I_{\{|y|<1\}} y \cdot \nabla_{x} \psi(x)\right] u(x+y) n^{m, \alpha}(d y) \\
& \quad-\int_{0<|y|<1} y \cdot \nabla_{x} \psi(x)[u(x+y)-u(x)] n^{m, \alpha}(d y) \\
= & :\left(I_{1} u\right)(x)+\left(I_{2} u\right)(x) .
\end{aligned}
$$

We estimate the $L^{p}$ norms of $I_{1} u$ and $I_{2} u$ in the last member.

First, rewrite $I_{1} u$ as

$$
\begin{gathered}
\left(I_{1} u\right)(x)=-\int_{0<|y|<1}\left[\psi(x+y)-\psi(x)-I_{\{|y|<1\}} y \cdot \nabla_{x} \psi(x)\right] u(x+y) n^{m, \alpha}(d y) \\
-\int_{|y| \geq 1}[\psi(x+y)-\psi(x)] u(x+y) n^{m, \alpha}(d y) .
\end{gathered}
$$

Hence

$\left|\left(I_{1} u\right)(x)\right| \leq\left\|\nabla^{2} \psi\right\|_{L^{\infty}} \int_{0<|y|<1}|y|^{2}|u(x+y)| n^{m, \alpha}(d y)+2\|\psi\|_{L^{\infty}} \int_{|y|>1}|u(x+y)| n^{m, \alpha}(d y)$,

so that for $1 \leq p<\infty$

$$
\left\|I_{1} u\right\|_{L^{p}}=\left(\int\left|\left(I_{1} u\right)(x)\right|^{p} d x\right)^{\frac{1}{p}} \leq\left(n_{1}^{m, \alpha}\left\|\nabla^{2} \psi\right\|_{L^{\infty}}+2 n_{\infty}^{m, \alpha}\|\psi\|_{L^{\infty}}\right)\|u\|_{L^{p}}
$$

where

$$
n_{\infty}^{m, \alpha}:=\int_{|y| \geq 1} n^{m, \alpha}(d y), \quad n_{\kappa}^{m, \alpha}:=\int_{0<|y|<1}|y|^{1+\kappa} n^{m, \alpha}(d y),
$$

where the former is finite, and the latter is finite for all $0<\kappa \leq 1$.

Next, for $I_{2} u$ we use the following known fact for an operator $T$ on $L^{p}\left(\mathbb{R}^{d}\right)$ with Calderón-Zygmund kernel $K: \mathbb{R}^{d} \backslash\{0\} \rightarrow \mathbb{C}$ (e.g. [St70, II.3, pp.35-42], [G10, Theorem 5.3.3, p.359], [MSc13, Def.7.1, Prop.7.4, Theorem 7.5, pp.166-172]). It is the integral kernel which satisfies, for some constant $B>0$, the following conditions:

(i) $|K(x)| \leq B|x|^{-d}$ for all $x \in \mathbb{R}^{d}$;

(ii) $\int_{|x| \geq 2|y|}|K(x)-K(x-y)| d x \leq B$ for all $y \neq 0$;

(iii) $\int_{R_{1}<|x|<R_{2}} K(x) d x=0$ for all $0<R_{1}<R_{2}<\infty$. 
Proposition 2.4. Let

$$
(T f)(x):=\lim _{\varepsilon \downarrow 0} \int_{|x-y| \geq \epsilon} K(x-y) f(y) d y .
$$

Then

$$
\begin{aligned}
& \|T f\|_{L^{p}} \leq C_{p}\|f\|_{L^{p}}, \\
& 1<p<\infty, \\
& \|T f\|_{L_{w}^{1}} \equiv \sup _{a>0} a\left|\left\{x \in \mathbb{R}^{d} ;|(T f)(x)|>a\right\}\right| \leq C_{1}\|f\|_{L^{1}}, \\
& p=1 \text {. }
\end{aligned}
$$

This proposition is going to be used in the proof of Lemma 2.3 (i).

We continue the proof of Lemma 2.3 (i). It still remains to deal with $I_{2} u$, which is rewritten as

$$
\left(I_{2} u\right)(x)=-\sum_{j=1}^{d} \lim _{\varepsilon \downarrow 0} \int_{\varepsilon \leq|y|<1} \partial_{x_{j}} \psi(x)\left(x_{j}-y_{j}\right) n^{m, \alpha}(x-y) u(y) d y .
$$

Here each $x_{j} \cdot n^{m, \alpha}(x), 1 \leq j \leq d$, is a Calderón-Zygmund kernel (see Appendix A, (A.2)), so that we have by Proposition 2.4 with $1 \leq p<\infty$ there exists a constant $C_{p}>0$ such that

$$
\begin{aligned}
\left\|I_{2} u\right\|_{L^{p}} & \leq C_{p}\|\nabla \psi\|_{L^{\infty}}\|u\|_{L^{p}}, \quad 1<p<\infty, \\
\left\|I_{2} u\right\|_{L_{w}^{1}} & =\sup _{a>0} a\left|\left\{x \in \mathbb{R}^{d} ;\left|\left(I_{2} u\right)(x)\right|>a\right\}\right| \leq C_{1}\|\nabla \psi\|_{L^{\infty}}\|u\|_{L^{1}}, \quad p=1 .
\end{aligned}
$$

Thus we obtain

$$
\begin{aligned}
\left\|\left[\left(H_{0, m}\right)^{\alpha}, \psi\right] u\right\|_{L^{p}} & \leq\left\|I_{1} u\right\|_{L^{p}}+\left\|I_{2} u\right\|_{L^{p}} \\
& \leq\left(n_{1}^{m, \alpha}\left\|\nabla^{2} \psi\right\|_{L^{\infty}}+2 n_{\infty}^{m, \alpha}\|\psi\|_{L^{\infty}}+C_{p}\|\nabla \psi\|_{L^{\infty}}\right)\|u\|_{L^{p}},
\end{aligned}
$$

showing (i) for $1<p<\infty$.

Next, for (ii) for $p=1$, we have

$$
\begin{aligned}
\left\|\left[\left(H_{0, m}\right)^{\alpha}, \psi\right] u\right\|_{L_{w}^{1}} & \leq 2\left(\left\|I_{1} u\right\|_{L_{w}^{1}}+\left\|I_{2} u\right\|_{L_{w}^{1}}\right) \leq 2\left\|I_{1} u\right\|_{L^{1}}+2\left\|I_{2} u\right\|_{L_{w}^{1}} \\
& \leq 2\left(n_{1}^{m, \alpha}\left\|\nabla^{2} \psi\right\|_{L^{\infty}}+2 n_{\infty}^{m, \alpha}\|\psi\|_{L^{\infty}}+C_{1}\|\nabla \psi\|_{L^{\infty}}\right)\|u\|_{L^{1}}
\end{aligned}
$$

because $\left\|I_{1} u\right\|_{L_{w}^{1}} \leq\left\|I_{1} u\right\|_{L^{1}}$. This shows (ii), ending the proof of Lemma 2.3.

When $A \in L_{\text {loc }}^{2}$, our selfadjoint operator $S:=(-i \nabla-A)^{2}+m^{2}$ originally is being defined as the selfadjoint operator in $L^{2}\left(\mathbb{R}^{d}\right)$ associated with the closed quadratic form (2.1). As already noted in the proof of Lemma 2.1 (i), it also makes sense as an operator in the spaces $L^{p}\left(\mathbb{R}^{d}\right), 1 \leq p<\infty$, referring to the result [Si79, Theorem 2.3] or [Si82, Sect. B13]) that the Schrödinger semigroup $e^{-t S}=e^{-t\left[(-i \nabla-A)^{2}+m^{2}\right]}$ satisfies

$$
\left|e^{-t\left[(-i \nabla-A)^{2}+m^{2}\right]} g\right| \leq e^{-t\left[-\Delta+m^{2}\right]}|g|
$$

pointwise for any $g \in L^{2}\left(\mathbb{R}^{d}\right)$. This yields that for $1 \leq p<\infty, e^{-t\left(H_{A, m}\right)^{2}}$ is a bounded operator of $L^{p}\left(\mathbb{R}^{d}\right)$ into itself for all $t>0$, which also is a contraction semigroup.

Thus, the fractional powers of $S$ such as $S^{\frac{\alpha}{2}}=\left(H_{A, m}\right)^{\alpha}$ in (2.3) equally make sense in $L^{p}\left(\mathbb{R}^{d}\right)$.

Now, we give two crucial lemmas, Lemmas 2.5 and 2.6. 
Lemma 2.5. Let $0<\alpha<1$ and assume that $A \in\left[L_{\mathrm{loc}}^{2}\left(\mathbb{R}^{d}\right)\right]^{d}$. Then: (i) if $u \in L^{2}\left(\mathbb{R}^{d}\right)$, one has for $\chi, \psi \in C_{0}^{\infty}\left(\mathbb{R}^{d}\right)$

$$
\begin{aligned}
\left\|\chi\left[\left(H_{0, m}\right)^{\alpha} \psi-\psi\left(H_{A, m}\right)^{\alpha}\right] u\right\|_{L^{1}} & \equiv\left\|\chi\left(\left[-\Delta+m^{2}\right]^{\frac{\alpha}{2}} \psi-\psi\left[(-i \nabla-A)^{2}+m^{2}\right]^{\frac{\alpha}{2}}\right) u\right\|_{L^{1}} \\
& \leq C_{\alpha, A, m, \chi, \psi}\|u\|_{L^{2}},
\end{aligned}
$$

where $C_{\alpha, A, \chi, \psi}$ is a constant which depends on $0<\alpha<1, A, m>0, \chi$ and $\psi$, and which tends to $\infty$ as $\alpha \uparrow 1$.

(ii) In particular, when $A=0$, (2.18) reads: if $u \in L^{2}\left(\mathbb{R}^{d}\right)$, one has

$$
\left\|\chi\left[\left(H_{0, m}\right)^{\alpha}, \psi\right] u\right\|_{L^{1}} \leq C_{\alpha, 0, m, \chi, \psi}\|u\|_{L^{2}}
$$

For $A=0$, inequality (2.19) appears more useful in comparison with (2.14), Lemma 2.3 .

Proof of Lemma 2.5.

(i) We have only to show (2.18) when $u \in C_{0}^{\infty}\left(\mathbb{R}^{d}\right)$, since $C_{0}^{\infty}\left(\mathbb{R}^{d}\right)$ is dense in $L^{2}\left(\mathbb{R}^{d}\right)$. Note then that $H_{0, m} u$ and $H_{A, m} u$ belong to $L^{2}\left(\mathbb{R}^{d}\right)$.

We use formula $(2.3)$ for $\left(H_{0, m}\right)^{\alpha}$ as well as $\left(H_{A, m}\right)^{\alpha}$ to calculate

$$
\begin{aligned}
& {\left[\left(H_{0, m}\right)^{\alpha} \psi-\psi\left(H_{A, m}\right)^{\alpha}\right] u } \\
= & \frac{1}{\Gamma\left(\frac{2-\alpha}{2}\right)} \int_{0}^{\infty} t^{-\frac{\alpha}{2}}\left[e^{-t\left(H_{0, m}\right)^{2}}\left(H_{0, m}\right)^{2} \psi-\psi\left(H_{A, m}\right)^{2} e^{-t\left(H_{A, m}\right)^{2}}\right] u d t \\
= & \frac{1}{\Gamma\left(\frac{2-\alpha}{2}\right)} \int_{0}^{\infty} d t t^{-\frac{\alpha}{2}}\left(-\frac{d}{d t}\right)\left[e^{-\theta t\left(H_{0, m}\right)^{2}} \psi e^{-(1-\theta) t\left(H_{A, m}\right)^{2}}\right]_{\theta=0}^{\theta=1} u \\
= & -\frac{1}{\Gamma\left(\frac{2-\alpha}{2}\right)} \int_{0}^{\infty} d t t^{-\frac{\alpha}{2}} \frac{d}{d t} \int_{0}^{1} d \theta \frac{d}{d \theta}\left[e^{-\theta t\left(H_{0, m}\right)^{2}} \psi e^{-(1-\theta) t\left(H_{A, m}\right)^{2}}\right] u \\
= & \frac{1}{\Gamma\left(\frac{2-\alpha}{2}\right)} \int_{0}^{\infty} d t t^{-\frac{\alpha}{2}} \\
& \quad \times \frac{d}{d t}\left(t \int_{0}^{1} d \theta\left[e^{-\theta t\left(H_{0, m}\right)^{2}}\left[\left(H_{0, m}\right)^{2} \psi-\psi\left(H_{A, m}\right)^{2}\right] e^{-(1-\theta) t\left(H_{A, m}\right)^{2}}\right] u\right) .
\end{aligned}
$$

Then by integration by parts,

$$
\begin{aligned}
& {\left[\left(H_{0, m}\right)^{\alpha} \psi-\psi\left(H_{A, m}\right)^{\alpha}\right] u} \\
& =\frac{1}{\Gamma\left(\frac{2-\alpha}{2}\right)}\left[t^{-\frac{\alpha}{2}+1} \int_{0}^{1}\left(e^{-\theta t\left(H_{0, m}\right)^{2}}\left[\left(H_{0, m}\right)^{2} \psi-\psi\left(H_{A, m}\right)^{2}\right] e^{-(1-\theta) t\left(H_{A, m}\right)^{2}}\right) u d \theta\right]_{t=0}^{t=\infty} \\
& +\frac{\alpha}{2 \Gamma\left(\frac{2-\alpha}{2}\right)} \int_{0}^{\infty} d t t^{-\frac{\alpha}{2}} \int_{0}^{1} d \theta\left(e^{-\theta t\left(H_{0, m}\right)^{2}}\left[\left(H_{0, m}\right)^{2} \psi-\psi\left(H_{A, m}\right)^{2}\right] e^{-(1-\theta) t\left(H_{A, m}\right)^{2}}\right) u \\
& =\frac{\alpha}{2 \Gamma\left(\frac{2-\alpha}{2}\right)} \int_{0}^{\infty} d t t^{-\frac{\alpha}{2}} \int_{0}^{1} d \theta\left(e^{-\theta t\left(H_{0, m}\right)^{2}}\left[\left(H_{0, m}\right)^{2} \psi-\psi\left(H_{A, m}\right)^{2}\right] e^{-(1-\theta) t\left(H_{A, m}\right)^{2}}\right) u .
\end{aligned}
$$

Here we make two observations related to (2.20). First for its second member, the boundary value at $t \rightarrow \infty$ of the first term also vanishes, because the part

$$
e^{-\theta t\left(H_{0, m}\right)^{2}}[\cdots] e^{-(1-\theta) t\left(H_{A, m}\right)^{2}}=e^{-\theta t\left(-\Delta+m^{2}\right)}[\cdots] e^{-(1-\theta) t\left[(-i \nabla-A)^{2}+m^{2}\right]}
$$


contains the factor $e^{-m^{2} t}$. Second for its last member, note that the middle factor in the integrand is, by (2.11) with $A:=0, B:=A$, equal to

$$
\left[\left(H_{0, m}\right)^{2} \psi-\psi\left(H_{A, m}\right)^{2}\right]=[i \nabla((i \nabla \psi)-\psi A)+((i \nabla \psi)-\psi A)(i \nabla+A)]
$$

as quadratic forms.

Substituting (2.21) into (2.20), we have with $\chi \in C_{0}^{\infty}\left(\mathbb{R}^{d}\right)$

$$
\begin{aligned}
\chi & {\left[\left(H_{0, m}\right)^{\alpha} \psi-\psi\left(H_{A, m}\right)^{\alpha}\right] u } \\
= & \frac{\alpha}{2 \Gamma\left(\frac{2-\alpha}{2}\right)} \int_{0}^{\infty} d t t^{-\frac{\alpha}{2}} \int_{0}^{1} d \theta \chi\left(e^{-\theta t\left(H_{0, m}\right)^{2}} i \nabla((i \nabla \psi)-\psi A) e^{-(1-\theta) t\left(H_{A, m}\right)^{2}}\right) u \\
& +\frac{\alpha}{2 \Gamma\left(\frac{2-\alpha}{2}\right)} \int_{0}^{\infty} d t t^{-\frac{\alpha}{2}} \int_{0}^{1} d \theta \chi\left(e^{-\theta t\left(H_{0, m}\right)^{2}}((i \nabla \psi)-\psi A)(i \nabla+A) e^{-(1-\theta) t\left(H_{A, m}\right)^{2}}\right) u \\
= & : I_{3} u+I_{4} u .
\end{aligned}
$$

We estimate the $L^{1}$ norm for $I_{3} u$ and $I_{4} u$ in (2.22). Note that $e^{-t(-i \nabla-A)^{2}}, t \geq 0$, is a contraction on $L^{p}\left(\mathbb{R}^{d}\right), 1 \leq p \leq \infty$.

First, for $I_{3} u$, integrate its absolute value in $x$ to get

$$
\begin{aligned}
\left\|I_{3} u\right\|_{L^{1}} \leq & \frac{\alpha}{2 \Gamma\left(\frac{2-\alpha}{2}\right)} \int_{0}^{\infty} t^{-\frac{\alpha}{2}} e^{-m^{2} t} d t \int_{0}^{1} d \theta \\
& \times\left\|\chi\left[e^{-\theta t(-\Delta)}(i \nabla)\right]((i \nabla \psi)-\psi A) e^{-(1-\theta) t(-i \nabla-A)^{2}} u\right\|_{L^{1}}
\end{aligned}
$$

Then by Lemma 2.1 (ii) for $p=1$, the Schwarz inequality and Lemma 2.1 (i)

$$
\begin{aligned}
\left\|I_{3} u\right\|_{L^{1}} & \leq \frac{\alpha}{2 \Gamma\left(\frac{2-\alpha}{2}\right)} \int_{0}^{\infty} t^{-\frac{\alpha}{2}} e^{-m^{2} t} d t \int_{0}^{1} d \theta \\
& \times\|\chi\|_{L^{\infty}}\left\|e^{-\theta t(-\Delta)}(i \nabla)\right\|_{\left[L^{1}\right]^{d} \rightarrow\left[L^{1}\right]^{d}}\left\|((i \nabla \psi)-\psi A) e^{-(1-\theta) t(-i \nabla-A)^{2}} u\right\|_{L^{1} \rightarrow\left[L^{1}\right]^{d}} \\
& \leq \frac{\alpha}{2 \Gamma\left(\frac{2-\alpha}{2}\right)} \int_{0}^{\infty} t^{-\frac{\alpha}{2}} e^{-m^{2} t} d t \int_{0}^{1} d \theta \\
& \times\|\chi\|_{L^{\infty}} C_{11}(\theta t)^{-1 / 2}\|(i \nabla \psi)-\psi A\|_{L^{2}}\left\|e^{-(1-\theta) t(-i \nabla-A)^{2}} u\right\|_{L^{2}} \\
& \leq \frac{C_{11} \alpha}{2 \Gamma\left(\frac{2-\alpha}{2}\right)} \int_{0}^{\infty} t^{-\frac{1+\alpha}{2}} e^{-m^{2} t} d t \int_{0}^{1} \frac{d \theta}{\theta^{1 / 2}}\|\chi\|_{L^{\infty}}\|(i \nabla \psi)-\psi A\|_{L^{2}}\|u\|_{L^{2}}
\end{aligned}
$$

Here recall that $\|(i \nabla \psi)-\psi A\|_{L^{2}}<\infty$ by assumption on $A$ and notice also that

$$
\int_{0}^{\infty} t^{-\frac{1+\alpha}{2}} e^{-m^{2} t} d t=\Gamma\left(\frac{1-\alpha}{2}\right) m^{-\frac{1-\alpha}{2}}
$$

which diverges as $\alpha \uparrow 1$ with $m>0$. Thus we have

$$
\left\|I_{3} u\right\|_{L^{1}} \leq \frac{C_{11} \alpha \Gamma\left(\frac{1-\alpha}{2}\right)}{\Gamma\left(\frac{2-\alpha}{2}\right) m^{\frac{1-\alpha}{2}}}\|(i \nabla \psi)-\psi A\|_{L^{2}}\|\chi\|_{L^{\infty}}\|u\|_{L^{2}} .
$$


Next for $I_{4} u$, in a similar way, we have from (2.22)

$$
\begin{gathered}
\left\|I_{4} u\right\|_{L^{1}} \leq \frac{\alpha}{2 \Gamma\left(\frac{2-\alpha}{2}\right)} \int_{0}^{\infty} t^{-\frac{\alpha}{2}} e^{-m^{2} t} d t \int_{0}^{1} d \theta \| \chi\left(e^{-\theta t(-\Delta)}((i \nabla \psi)-\psi A)\right. \\
\left.\times\left[(i \nabla+A) e^{-(1-\theta) t(-i \nabla-A)^{2}}\right]\right) u \|_{L^{1}}
\end{gathered}
$$

Then by the Schwarz inequality and Lemma 2.1 (iv)

$$
\begin{aligned}
\left\|I_{4} u\right\|_{L^{1}} \leq & \frac{\alpha}{2 \Gamma\left(\frac{2-\alpha}{2}\right)} \int_{0}^{\infty} t^{-\frac{\alpha}{2}} e^{-m^{2} t} d t \int_{0}^{1} d \theta\|\chi\|_{L^{\infty}}\left\|e^{-\theta t(-\Delta)}\right\|_{L^{1} \rightarrow L^{1}} \\
& \left.\times \|((i \nabla \psi)-\psi A)\left[(i \nabla+A) e^{-(1-\theta) t(-i \nabla-A)^{2}}\right]\right) u \|_{L^{1}} \\
\leq & \frac{\alpha}{2 \Gamma\left(\frac{2-\alpha}{2}\right)} \int_{0}^{\infty} t^{-\frac{\alpha}{2}} e^{-m^{2} t} d t \int_{0}^{1} d \theta\|\chi\|_{L^{\infty}}\left\|e^{-\theta t(-\Delta)}\right\|_{L^{1} \rightarrow L^{1}}\|(i \nabla \psi)-\psi A\|_{L^{2}} \\
& \left.\times \|(i \nabla+A) e^{-(1-\theta) t(-i \nabla-A)^{2}}\right] u \|_{L^{2}} \\
\leq & \frac{\alpha}{2 \Gamma\left(\frac{2-\alpha}{2}\right)} \int_{0}^{\infty} t^{-\frac{\alpha}{2}} e^{-m^{2} t} d t \int_{0}^{1} d \theta\|\chi\|_{L^{\infty}}\|(i \nabla \psi)-\psi A\|_{L^{2}}\left(\frac{d}{2 e(1-\theta)}\right)^{1 / 2}\|u\|_{L^{2}} \\
= & \left(\frac{d}{2 e}\right)^{1 / 2} \frac{\alpha}{2 \Gamma\left(\frac{2-\alpha}{2}\right)} \int_{0}^{\infty} t^{-\frac{1+\alpha}{2}} e^{-m^{2} t} d t \int_{0}^{1} \frac{d \theta}{(1-\theta)^{1 / 2}}\|\chi\|_{L^{\infty}}\|(i \nabla \psi)-\psi A\|_{L^{2}}\|u\|_{L^{2}} .
\end{aligned}
$$

Then we have

$$
\left\|I_{4} u\right\|_{L^{1}} \leq\left(\frac{d}{2 e}\right)^{1 / 2} \frac{\alpha \Gamma\left(\frac{1-\alpha}{2}\right)}{\Gamma\left(\frac{2-\alpha}{2}\right) m^{\frac{1-\alpha}{2}}}\|(i \nabla \psi)-\psi A\|_{L^{2}}\|\chi\|_{L^{\infty}}\|\chi\|_{L^{\infty}}\|u\|_{L^{2}} .
$$

Putting (2.24) and (2.26) together in view of (2.22), we have

$$
\begin{aligned}
& \left\|\chi\left[\left(H_{0, m}\right)^{\alpha} \psi-\psi\left(H_{A, m}\right)^{\alpha}\right] u\right\|_{L^{1}} \\
& \leq 2\left(\left\|I_{3} u\right\|_{L^{1}}+\left\|I_{4} u\right\|_{L^{1}}\right) \\
& \leq 2 \frac{C_{11}+\left(\frac{d}{2 e}\right)^{1 / 2}}{m^{\frac{1-\alpha}{2}}} \frac{\alpha \Gamma\left(\frac{1-\alpha}{2}\right)}{\Gamma\left(\frac{2-\alpha}{2}\right)}\|(i \nabla \psi)-\psi A\|_{L^{2}}\|\chi\|_{L^{\infty}}\|u\|_{L^{2}} .
\end{aligned}
$$

This yields (2.18), showing Lemma 2.5 (i).

(ii) Inequality (2.19) is immediately derived by putting $A=0$ in (2.18).

This shows Lemma 2.5 (ii), completing the proof of Lemma 2.5.

From Lemma 2.5 we have the following result which we shall need, in particular, assertion (ii), in the proof of Theorem 1.1.

Lemma 2.6. Let $0<\alpha<1$. Assume that $A \in\left[L_{\mathrm{loc}}^{2}\left(\mathbb{R}^{d}\right)\right]^{d}$.

(i) If $u \in\left(C^{\infty} \cap L^{2}\right)\left(\mathbb{R}^{d}\right)$, then $\left(H_{A, m}\right)^{\alpha} u$ is locally in $L^{1}\left(\mathbb{R}^{d}\right)$.

(ii) If $u \in L^{2}\left(\mathbb{R}^{d}\right)$ with $\left(H_{A, m}\right)^{\alpha} u \in L_{\text {loc }}^{1}\left(\mathbb{R}^{d}\right)$, then $\left(H_{0, m}\right)^{\alpha} u$ is locally in $L^{1}\left(\mathbb{R}^{d}\right)$.

Proof. (i) Let $u \in\left(C^{\infty} \cap L^{2}\right)\left(\mathbb{R}^{d}\right)$. Then for $\psi \in C_{0}^{\infty}\left(\mathbb{R}^{d}\right)$,

$$
\psi\left(H_{A, m}\right)^{\alpha} u=\left(H_{0, m}\right)^{\alpha}(\psi u)+\left(\psi\left(H_{A, m}\right)^{\alpha}-\left(H_{0, m}\right)^{\alpha} \psi\right) u
$$


Put $K=\operatorname{supp} \psi$. Then, since $\psi u$ is in $C_{0}^{\infty}\left(\mathbb{R}^{d}\right)$, the first term $\left(H_{0, m}\right)^{\alpha}(\psi u)$ on the right-hand side belongs to $L^{2}\left(\mathbb{R}^{d}\right)$, as we can see from (2.6) (with $\psi u$ instead of $u$ ) or Lemma $2.2(2.4)$ with $A=0$ (with $\psi u$ instead of $\varphi$ ). For the second term restricted to $K$, it belongs to $L^{1}(K)$, as we see by Lemma $2.5(2.18)$. Therefore $\psi\left(H_{A, m}\right)^{\alpha} u$ is in $L^{1}(K)$, so that $\left(H_{A, m}\right)^{\alpha} u$ is locally in $L^{1}\left(\mathbb{R}^{d}\right)$. This proves the assertion (i).

(ii) Let $u \in L^{2}$ with $\left(H_{A, m}\right)^{\alpha} u \in L_{\text {loc }}^{1}$ and let $K$ be an arbitrary compact subset of $\mathbb{R}^{d}$. Take $\chi, \psi \in C_{0}^{\infty}\left(\mathbb{R}^{d}\right)$ with $0 \leq \chi(x) \leq 1$ such that $\chi(x)=\psi(x)=1$ on $K$. Then since

$$
\psi\left(H_{0, m}\right)^{\alpha} u-\psi\left(H_{A, m}\right)^{\alpha} u=-\left[\left(H_{0, m}\right)^{\alpha}, \psi\right] u+\left(\left(H_{0, m}\right)^{\alpha} \psi-\psi\left(H_{A, m}\right)^{\alpha}\right) u,
$$

we have by Lemma $2.5(2.18)$ with $A=0$ as well as with non-zero $A$

$$
\begin{aligned}
& \left\|\left(H_{0, m}\right)^{\alpha} u-\psi\left(H_{A, m}\right)^{\alpha} u\right\|_{L^{1}(K)} \\
& =\left\|\chi \psi\left[\left(H_{0, m}\right)^{\alpha} u-\left(H_{A, m}\right)^{\alpha} u\right]\right\|_{L^{1}(K)} \\
& \leq\left\|\chi\left[\left(H_{0, m}\right)^{\alpha}, \psi\right] u\right\|_{L^{1}}+\left\|\chi\left(\left(H_{0, m}\right)^{\alpha} \psi-\psi\left(H_{A, m}\right)^{\alpha}\right) u\right\|_{L^{1}} \\
& \leq\left(C_{\alpha, 0, m, \chi, \psi}+C_{\alpha, A, m, \chi, \psi}\right)\|u\|_{L^{2}}<\infty .
\end{aligned}
$$

Since by assumption $\left(H_{A, m}\right)^{\alpha} u$ is locally in $L^{1}\left(\mathbb{R}^{d}\right)$, we have $\left(H_{0, m}\right)^{\alpha} u$ is locally $L^{1}\left(\mathbb{R}^{d}\right)$. This proves the assertion (ii), ending the proof of Lemma 2.6.

\section{Proof of Theorems}

We show only Theorem 1.1 and Theorem 1.2. As for Theorem 1.3, essential selfadjointness of $H_{A, V, m}$ follows from Theorem 1.1 by its standard application in Kato's original paper [K72]. In fact, one can show in the same way as in [I89, Theorem 5.1]. So the proof is omitted. The assertion that $H_{A, V, m}=H_{A, m}+V \geq m$ is trivial because $H_{A, m} \geq m$.

In this section, we keep assuming that $m>0$ before come to the final part (iii) of the proof of Theorem 1.1.

\subsection{Proof of Theorem 1.1}

The proof will proceed similarly to Kato's original proof [K72] (e.g. [ReSi75, Theorems X.27 (p.183), X.33 (p.188)]) for the magnetic nonrelativistic Schrödinger operator $\frac{1}{2 m}(-i \nabla-A(x))^{2}$ and to a modified one [I89], [ITs92] for another magnetic relativistic Schrödinger operator. However, if one could show the assumption of the theorem that $u \in L^{2}$ with $H_{A, m} u \in L_{\text {loc }}^{1}$ implies that $\partial_{j} u \in L_{\text {loc }}^{1}, 1 \leq j \leq d$, and/or $H_{0, m} u \in L_{\text {loc }}^{1}$, there should be no problem. The obstruction seems to come from the fact that the operators $\partial_{j} \cdot\left(-\Delta+m^{2}\right)^{-1 / 2}, 1 \leq j \leq d$, are not bounded from $L^{1}$ to $L^{1}$, though they are bounded from $L^{1}$ to $L_{w}^{1}$. The strategy we adopt to cope with this difficulty is, in the beginning, to make a detour by considering the case $\left(H_{A, m}\right)^{\alpha}$ for $\alpha<1$, leaving the very case $\alpha=1$ aside, however, to handle the local convergence in $L^{1}$. In fact, in the first stage (Lemmas 3.1 and 3.2), we show first that if $\left(H_{A, m}\right)^{\alpha} u \in L_{\text {loc }}^{1}$, then $\left(H_{A, m}\right)^{\alpha} u^{\delta} \rightarrow\left(H_{A, m}\right)^{\alpha} u$ locally in $L^{1}$ as $\delta \downarrow 0$, and making use of Lemma 2.6 saying that $\left(H_{0, m}\right)^{\alpha} u$ is locally in $L^{1}$. Next we show that the assumption $H_{A, m} u \in L_{\text {loc }}^{1}$ implies that $\left(H_{A, m}\right)^{\alpha} u \in L_{\mathrm{loc}}^{1}$ for $0<\alpha<1$, and $\left(H_{A, m}\right)^{\alpha} u$ converges to $H_{A, m} u$ in $L_{\mathrm{loc}}^{1}$ 
as $\alpha \uparrow 1$. In the second, main stage, with $m>0$, we show first for $0<\alpha<1$ that the asserted inequality, i.e.

$$
\operatorname{Re}\left((\operatorname{sgn} u)\left[\left(H_{A, m}\right)^{\alpha}-m^{\alpha}\right] u\right) \geq\left[\left(H_{0, m}\right)^{\alpha}-m^{\alpha}\right]|u|,
$$

holds, and next for $\alpha=1$, using the just above mentioned fact that $\left(H_{A, m}\right)^{\alpha} u \rightarrow H_{A, m} u$ in $L_{\text {loc }}^{1}$ as $\alpha \uparrow 1$. The final stage will deal with the remaining case for $m=0$ and $\alpha=1$.

We provide two lemmas playing a crucial role in the proof of Theorem 1.1.

For a function $f$ locally in $L^{1}\left(\mathbb{R}^{d}\right)$, we write its mollifier as $f^{\delta}=\rho_{\delta} * f, 0<\delta \leq 1$, where $\rho_{\delta}(x):=\delta^{-d} \rho(x / \delta)$, and $\rho(x)$ is a nonnegative $C^{\infty}$ function $\mathbb{R}^{d}$ with compact support $\operatorname{supp} \rho \subseteq\{x ;|x| \leq 1\}$ and $\int \rho(x) d x=1$.

Lemma 3.1. Let $0<\alpha<1$. Let $u \in L^{2}\left(\mathbb{R}^{d}\right)$, so that $u^{\delta}:=\rho_{\delta} * u \rightarrow u$ in $L^{2}$ as $\delta \downarrow 0$. If $\left(H_{A, m}\right)^{\alpha} u \in L_{\mathrm{loc}}^{1}\left(\mathbb{R}^{d}\right)$, then $\left(H_{A, m}\right)^{\alpha} u^{\delta}=\left[(-i \nabla-A)^{2}+m^{2}\right]^{\alpha / 2} u^{\delta} \rightarrow\left(H_{A, m}\right)^{\alpha} u=$ $\left[(-i \nabla-A)^{2}+m^{2}\right]^{\alpha / 2} u$ locally in $L^{1}\left(\mathbb{R}^{d}\right)$ as $\delta \downarrow 0$.

Proof. Let $u \in L^{2}$ and $\left(H_{A, m}\right)^{\alpha} u \in L_{\text {loc }}^{1}\left(\mathbb{R}^{d}\right)$. Then by Lemma 2.6 (ii), $\left(H_{0, m}\right)^{\alpha} u$ is locally in $L^{1}$ and since $u^{\delta} \in C^{\infty} \cap L^{2}$, we have by Lemma 2.6 (i) that $\left(H_{A, m}\right)^{\alpha} u^{\delta}$ is locally in $L^{1}$. The important is: thanks to the integral operator representation (2.6) of the operator $\left(H_{0, m}\right)^{\alpha}$, the convolution commutes with $\left(H_{0, m}\right)^{\alpha}$. Therefore we have $\left(\left(H_{0, m}\right)^{\alpha} u\right)^{\delta}=\left(H_{0, m}\right)^{\alpha} u^{\delta}$, which converges to $\left(H_{0, m}\right)^{\alpha} u$ locally in $L^{1}$ as $\delta \downarrow 0$. Then for $K$ a compact subset in $\mathbb{R}^{d}$, let $\chi, \psi \in C_{0}^{\infty}\left(\mathbb{R}^{d}\right)$ with $0 \leq \chi(x) \leq 1$ on $\mathbb{R}^{d}$ and $\chi(x)=\psi(x)=1$ on $K$. We have

$$
\begin{aligned}
& \left\|\left(H_{A, m}\right)^{\alpha} u^{\delta}-\left(H_{A, m}\right)^{\alpha} u\right\|_{L^{1}(K)} \\
& =\left\|\chi \psi\left(H_{A, m}\right)^{\alpha}\left(u^{\delta}-u\right)\right\|_{L^{1}(K)} \\
& =\left\|\chi\left[-\left(H_{0, m}\right)^{\alpha} \psi+\left(\left(H_{0, m}\right)^{\alpha} \psi-\psi\left(H_{A, m}\right)^{\alpha}\right)\right]\left(u^{\delta}-u\right)\right\|_{L^{1}(K)} \\
& \leq\left\|\chi\left(H_{0, m}\right)^{\alpha} \psi\left(u^{\delta}-u\right)\right\|_{L^{1}}+\left\|\chi\left[\left(H_{0, m}\right)^{\alpha} \psi-\psi\left(H_{A, m}\right)^{\alpha}\right]\left(u^{\delta}-u\right)\right\|_{L^{1}} .
\end{aligned}
$$

The second term in the last member of the above inequality is, by Lemma 2.5 (2.18), estimated from above by $C_{\alpha, A, m, \chi, \psi}\left\|u^{\delta}-u\right\|_{L^{2}}$. The first term is equal to

$$
\begin{aligned}
& \left\|\chi\left(\left[\left(H_{0, m}\right)^{\alpha}, \psi\right]+\psi\left(H_{0, m}\right)^{\alpha}\right)\left(u^{\delta}-u\right)\right\|_{L^{1}} \\
& \leq\left\|\chi\left[\left(H_{0, m}\right)^{\alpha}, \psi\right]\left(u^{\delta}-u\right)\right\|_{L^{1}}+\left\|\chi \psi\left[\left(H_{0, m}\right)^{\alpha} u^{\delta}-\left(H_{0, m}\right)^{\alpha} u\right]\right\|_{L^{1}} \\
& \leq C_{\alpha, 0, m, \chi, \psi}\left\|u^{\delta}-u\right\|_{L^{2}}+\left\|\left(\left(H_{0, m}\right)^{\alpha} u\right)^{\delta}-\left(H_{0, m}\right)^{\alpha} u\right\|_{L^{1}},
\end{aligned}
$$

where we have used for the first term Lemma 2.5 (2.19) for $A=0$ and for the second the fact that $\left(H_{0, m}\right)^{\alpha} u^{\delta}=\left(\left(H_{0, m}\right)^{\alpha} u\right)^{\delta}$ since by assumption, $\left(H_{0, m}\right)^{\alpha} u$ is locally in $L^{1}$ and $u \in L^{2}$. It follows that

$$
\begin{aligned}
& \left\|\left(H_{A, m}\right)^{\alpha} u^{\delta}-\left(H_{A, m}\right)^{\alpha} u\right\|_{L^{1}(K)} \\
& \leq C_{\alpha, 0, m, \chi, \psi}\left\|u^{\delta}-u\right\|_{L^{2}}+\|\psi\|_{L^{\infty}}\left\|\left(\left(H_{0, m}\right)^{\alpha} u\right)^{\delta}-\left(H_{0, m}\right)^{\alpha} u\right\|_{L^{1}}+C_{\alpha, A, m, \chi, \psi}\left\|u^{\delta}-u\right\|_{L^{2}},
\end{aligned}
$$

which approaches zero as $\delta \downarrow 0$. This proves Lemma 3.1.

Lemma 3.2. Let $0<\alpha \leq 1$. Let $u \in L^{2}\left(\mathbb{R}^{d}\right)$ and $H_{A, m} u \in L_{\text {loc }}^{1}\left(\mathbb{R}^{d}\right)$. Then $\left(H_{A, m}\right)^{\alpha} u=\left[(-i \nabla-A)^{2}+m^{2}\right]^{\alpha / 2} u$ is also in $L_{\text {loc }}^{1}\left(\mathbb{R}^{d}\right)$, and $\left\{\left(H_{A, m}\right)^{\alpha} u\right\}$ converges to $H_{A, m} u$ in $L_{\text {loc }}^{1}\left(\mathbb{R}^{d}\right)$ as $\alpha \uparrow 1$. Namely, for any $\psi \in C_{0}^{\infty}\left(\mathbb{R}^{d}\right),\left\|\psi\left(H_{A, m}\right)^{\alpha} u\right\|_{L^{1}}$ is uniformly bounded for $0<\alpha \leq 1$, and $\left\{\psi\left(H_{A, m}\right)^{\alpha} u\right\}$ converges to $\psi H_{A, m} u$ in $L^{1}\left(\mathbb{R}^{d}\right)$ as $\alpha \uparrow 1$. 
Proof. Let $0<\alpha<1$. To begin with, suppose with $\psi \in C_{0}^{\infty}\left(\mathbb{R}^{d}\right)$ that some $u \in L^{2}\left(\mathbb{R}^{d}\right)$ satisfies the equality

$$
\psi\left(H_{A, m}\right)^{\alpha} u=\left(H_{A, m}\right)^{-(1-\alpha)} \psi H_{A, m} u+\left[\psi,\left(H_{A, m}\right)^{-(1-\alpha)}\right] H_{A, m} u .
$$

This holds at least if $u \in D\left[H_{A, m}\right]$, and hence, in particular, if $u=\phi \in C_{0}^{\infty}\left(\mathbb{R}^{d}\right)$. Note here that $\left(H_{A, m}\right)^{\alpha}$ has $D\left[H_{A, m}\right]$ as an operator core, while $H_{A, m}$ has $C_{0}^{\infty}\left(\mathbb{R}^{d}\right)$ as an operator core.

Now, let $u \in L^{2}\left(\mathbb{R}^{d}\right)$ with $H_{A, m} u \in L_{\text {loc }}^{1}\left(\mathbb{R}^{d}\right)$, just what is assumed by Lemma 3.2. The first term on the right-hand side of $(3.2)$ is in $L^{1}\left(\mathbb{R}^{d}\right)$, since by Lemma 2.1 (i) with $p=1,\left(H_{A, m}\right)^{-(1-\alpha)}$ is a bounded operator which is a contraction mapping $L^{1}\left(\mathbb{R}^{d}\right)$ into $L^{1}\left(\mathbb{R}^{d}\right)$, bounded uniformly for $0<\alpha \leq 1$ and strongly continuous there, so long as $m>0$. The term on the left-hand side of (3.2) exists as a distribution. The second term on the right-hand side lies in the dual space of the space $D\left[H_{A, m}\right]$, considered as a Hilbert space equipped with the graph norm $\|v\|_{L^{2}}^{2}+\left\|H_{A, m} v\right\|^{2}$. Here recall (2.1) and note that for $\phi \in C_{0}^{\infty}\left(\mathbb{R}^{d}\right)$

$$
\left\|\left(H_{A, m}\right)^{\alpha} \phi\right\|_{L^{2}}=\left\|\left(H_{A, m}\right)^{-(1-\alpha)} H_{A, m} \phi\right\|_{L^{2}} \leq\left\|H_{A, m} \phi\right\|_{L^{2}} .
$$

Thus all the three terms on the left- and right-hand sides of (3.2) exist also as distributions.

To show the assertion of the lemma, take a $C^{\infty}$ cutoff function $\chi$ with compact support, a similar one of which has already been used before, such that $0 \leq \chi(x) \leq 1$ in $\mathbb{R}^{d}$ with $\chi(x)=1$ on $\operatorname{supp} \psi$. As $\psi=\chi \psi$ holds, so does $\psi\left(H_{A, m}\right)^{\alpha} u=\chi \psi\left(H_{A, m}\right)^{\alpha} u$. Then consider the (3.2) multiplied by $\chi$, i.e.

$$
\psi\left(H_{A, m}\right)^{\alpha} u=\chi\left(H_{A, m}\right)^{-(1-\alpha)} \psi H_{A, m} u+\chi\left[\psi,\left(H_{A, m}\right)^{-(1-\alpha)}\right] H_{A, m} u .
$$

The first term on the right of (3.2) (and hence (3.3)) converges to $\psi H_{A, m} u$ as $\alpha \uparrow 1$, since $\left(H_{A, m}\right)^{-(1-\alpha)}$ is an operator on $L^{1}\left(\mathbb{R}^{d}\right)$, bounded uniformly for $0<\alpha \leq 1$ and strongly continuous there, so long as $m>0$. So we have only to show the second term of (3.3), i.e. $\chi\left[\psi,\left(H_{A, m}\right)^{-(1-\alpha)}\right] H_{A, m} u$ lie in $L^{1}\left(\mathbb{R}^{d}\right)$, being uniformly bounded, and converge to 0 in $L^{1}$ as $\alpha \uparrow 1$.

Use formula (2.2) to rewrite this second term on the right of (3.3) as

$$
\begin{aligned}
& \chi\left[\psi,\left(H_{A, m}\right)^{-(1-\alpha)}\right] H_{A, m} u \\
& =\frac{1}{\Gamma\left(\frac{1-\alpha}{2}\right)} \int_{0}^{\infty} d t t^{\frac{1-\alpha}{2}-1} \chi\left[\psi e^{-t\left(H_{A, m}\right)^{2}}-e^{-t\left(H_{A, m}\right)^{2}} \psi\right] H_{A, m} u \\
& =-\frac{1}{\Gamma\left(\frac{1-\alpha}{2}\right)} \int_{0}^{\infty} d t t^{-\frac{1+\alpha}{2}} \chi \int_{0}^{1} d \theta \frac{d}{d \theta}\left[e^{-\theta t\left(H_{A, m}\right)^{2}} \psi e^{-(1-\theta) t\left(H_{A, m}\right)^{2}}\right] H_{A, m} u \\
& =\frac{1}{\Gamma\left(\frac{1-\alpha}{2}\right)} \int_{0}^{\infty} d t t^{\frac{1-\alpha}{2}}\left[\left(H_{A, m}\right)^{2}, \psi\right] e^{-(1-\theta) t\left(H_{A, m}\right)^{2}} H_{A, m} u .
\end{aligned}
$$

Recall identity (2.10) for the commutator $\left[\left(H_{A, m}\right)^{2}, \psi\right]$, indeed, the first of the two expressions there and substitute it into the $\left[\left(H_{A, m}\right)^{2}, \psi\right]$ in the last member of (3.4). 
Then

$$
\begin{aligned}
\chi & {\left[\psi,\left(H_{A, m}\right)^{-(1-\alpha)}\right] H_{A, m} u } \\
= & \frac{1}{\Gamma\left(\frac{1-\alpha}{2}\right)} \int_{0}^{\infty} d t t^{\frac{1-\alpha}{2}} \int_{0}^{1} d \theta \chi e^{-\theta t\left(H_{A, m}\right)^{2}}(\Delta \psi) e^{-(1-\theta) t\left(H_{A, m}\right)^{2}} H_{A, m} u \\
& +\frac{2}{\Gamma\left(\frac{1-\alpha}{2}\right)} \int_{0}^{\infty} d t t^{\frac{1-\alpha}{2}} \int_{0}^{1} d \theta \chi e^{-\theta t\left(H_{A, m}\right)^{2}}(i \nabla+A)(i \nabla \psi) e^{-(1-\theta) t\left(H_{A, m}\right)^{2}} H_{A, m} u \\
= & : I_{5} u+I_{6} u .
\end{aligned}
$$

We estimate the $L^{1}$ norms of $I_{5} u$ and $I_{6} u$ in (3.5).

First for $I_{5} u$, integrate its absolute value in $x$, we have by the Schwarz inequality

$$
\begin{aligned}
&\left\|I_{5} u\right\|_{L^{1}} \leq \frac{1}{\Gamma\left(\frac{1-\alpha}{2}\right)} \int_{0}^{\infty} d t t^{\frac{1-\alpha}{2}} \int_{0}^{1} d \theta\left\|\chi e^{-\theta t\left(H_{A, m}\right)^{2}}(\Delta \psi) e^{-(1-\theta) t\left(H_{A, m}\right)^{2}} H_{A, m} u\right\|_{L^{1}} \\
& \leq \frac{1}{\Gamma\left(\frac{1-\alpha}{2}\right)} \int_{0}^{\infty} d t t^{\frac{1-\alpha}{2}} \int_{0}^{1} d \theta\|\chi\|_{L^{2}} \\
& \leq \quad \times\left\|e^{-\theta t\left(H_{A, m}\right)^{2}}(\Delta \psi) e^{-(1-\theta) t\left(H_{A, m}\right)^{2}} H_{A, m} u\right\|_{L^{2}} \\
& \Gamma \quad \times\|\Delta \psi\|_{L^{\infty}}\left\|e^{-(1-\theta) t\left(H_{A, m}\right)^{2}} H_{A, m}\right\|_{L^{2} \rightarrow L^{2}}\|u\|_{L^{2}} \\
& \leq \frac{1}{\Gamma\left(\frac{1-\alpha}{2}\right)} \int_{0}^{\infty} d t t^{\frac{1-\alpha}{2}} \int_{0}^{1} d \theta\|\chi\|_{L^{2}}\left\|e^{-\theta t\left(H_{A, m}\right)^{2}}\right\|_{L^{2} \rightarrow L^{2}} d \theta\|\chi\|_{L^{2}} e^{-\frac{m^{2}}{2} \theta t}\left\|e^{-\frac{\theta t}{2}\left(H_{A, m}\right)^{2}}\right\|_{L^{2} \rightarrow L^{2}} \\
& \quad \times\|\Delta \psi\|_{L^{\infty}} e^{-\frac{m^{2}}{2}(1-\theta) t}\left\|e^{-\frac{(1-\theta) t}{2}\left(H_{A, m}\right)^{2}} H_{A, m}\right\|_{L^{2} \rightarrow L^{2}}\|u\|_{L^{2}} .
\end{aligned}
$$

Then by Lemma 2.1 (iii) we have the bound

$$
\begin{aligned}
\left\|I_{5} u\right\|_{L^{1}} & \leq \frac{1}{\Gamma\left(\frac{1-\alpha}{2}\right)} \int_{0}^{\infty} d t t^{\frac{1-\alpha}{2}} e^{-\frac{m^{2}}{2} t} \int_{0}^{1} d \theta\|\chi\|_{L^{2}}\|\Delta \psi\|_{L^{\infty}}\left(\frac{1}{2 e\left(\frac{1-\theta}{2}\right) t}\right)^{1 / 2}\|u\|_{L^{2}} \\
& \leq \frac{1}{\Gamma\left(\frac{1-\alpha}{2}\right)} \int_{0}^{\infty} d t t^{-\frac{\alpha}{2}} e^{-\frac{m^{2}}{2} t}(2 e)^{-1 / 2} \int_{0}^{1} \frac{d \theta}{\left(\frac{1-\theta}{2}\right)^{1 / 2}}\|\chi\|_{L^{2}}\|\Delta \psi\|_{L^{\infty}}\|u\|_{L^{2}} \\
& \leq 2^{\frac{3}{2}(2 e)^{-1 / 2}} \frac{\Gamma\left(\frac{2-\alpha}{2}\right)}{\Gamma\left(\frac{1-\alpha}{2}\right)}\left(\frac{2}{m^{2}}\right)^{\frac{2-\alpha}{2}}\|\chi\|_{L^{2}}\|\Delta \psi\|_{L^{\infty}}\|u\|_{L^{2}}
\end{aligned}
$$

Next for $I_{6} u$, we are going to show a similar bound

$$
\left\|I_{6} u\right\|_{L^{1}} \leq 4 \pi(2 e)^{-1 / 2} \frac{\Gamma\left(\frac{2-\alpha}{2}\right)}{\Gamma\left(\frac{1-\alpha}{2}\right)}\left(\frac{2}{m^{2}}\right)^{\frac{2-\alpha}{2}} C_{\chi, A}\|\nabla \psi\|_{L^{\infty}}\|u\|_{L^{2}},
$$

with a constant, depending only on $\chi$ and $A$,

$$
C_{\chi, A}:=\left[\|\nabla \chi\|_{L^{2}}^{2}+m^{2}\|\chi\|_{L^{2}}^{2}+\|\chi A\|_{L^{2}}^{2}\right]^{1 / 2},
$$

which is bounded since $A \in L_{\text {loc }}^{2}\left(\mathbb{R}^{d}\right)$. The proof is to integrate the absolute value of $I_{6} u$ in $x$ to get

$$
\left\|I_{6} u\right\|_{L^{1}} \leq \frac{2}{\Gamma\left(\frac{1-\alpha}{2}\right)} \int_{0}^{\infty} d t t^{\frac{1-\alpha}{2}} \int_{0}^{1} d \theta X_{A, m}(t, \theta ; \chi, \psi, u),
$$


where we put

$$
X_{A, m}(t, \theta ; \chi, \psi, u)=\left\|\chi e^{-\theta t\left(H_{A, m}\right)^{2}}(i \nabla+A)(i \nabla \psi) e^{-(1-\theta) t\left(H_{A, m}\right)^{2}} H_{A, m} u\right\|_{L^{1}} .
$$

Somewhat crucial is the estimate of $X_{A, m}(t, \theta ; \chi, \psi, u)$ in (3.10) which we are going to do, where the parentheses $(\cdot, \cdot)$ below stand for the $L^{2}$ inner product:

$$
\begin{aligned}
& X_{A, m}(t, \theta ; \chi, \psi, u) \\
& =\left|\left(\chi, e^{-\theta t\left(H_{A, m}\right)^{2}}(i \nabla+A)(i \nabla \psi) e^{-(1-\theta) t\left(H_{A, m}\right)^{2}} H_{A, m} u\right)\right| \\
& =\left|\left(\chi, e^{-\theta t\left(H_{A, m}\right)^{2}} H_{A, m} \cdot\left(H_{A, m}\right)^{-1}(i \nabla+A)(i \nabla \psi) e^{-(1-\theta) t\left(H_{A, m}\right)^{2}} H_{A, m} u\right)\right| \\
& =\left|\left(e^{-\theta t\left(H_{A, m}\right)^{2}} H_{A, m} \chi,\left(H_{A, m}\right)^{-1}(i \nabla+A)(i \nabla \psi) e^{-(1-\theta) t\left(H_{A, m}\right)^{2}} H_{A, m} u\right)\right| \\
& \leq\left\|e^{-\theta t\left(H_{A, m}\right)^{2}} H_{A, m} \chi\right\|_{L^{2}}\left\|\left(H_{A, m}\right)^{-1}(i \nabla+A)(i \nabla \psi) e^{-(1-\theta) t\left(H_{A, m}\right)^{2}} H_{A, m} u\right\|_{L^{2}} .
\end{aligned}
$$

In the last member of (3.11), the first factor and the second are estimated as follows:

$$
\begin{aligned}
& \left\|e^{-\theta t\left(H_{A, m}\right)^{2}} H_{A, m} \chi\right\|_{L^{2}} \leq e^{-m^{2} \theta t}\left\|H_{A, m} \chi\right\|_{L^{2}} \\
& \quad=e^{-m^{2} \theta t}\left[\sum_{j=1}^{d}\left\|\left(i \partial_{j}+A_{j}\right) \chi\right\|_{L^{2}}^{2}+m^{2}\|\chi\|_{L^{2}}^{2}\right]^{1 / 2} \\
& \leq e^{-m^{2} \theta t}\left[\|\nabla \chi\|_{L^{2}}^{2}+\|\chi A\|_{L^{2}}^{2}+m^{2}\|\chi\|_{L^{2}}^{2}\right]^{1 / 2} \\
& =e^{-m^{2} \theta t} C_{\chi, A}
\end{aligned}
$$

Here in (3.12) and (3.13) we have used (2.1), Lemma 2.1 (iii), and the estimate $\left\|\left(H_{A, m}\right)^{-1}(i \nabla+A)\right\|_{L^{2} \rightarrow L^{2}} \leq 1$. From (3.12) and (3.13) we obtain

$$
\begin{aligned}
\left\|I_{6} u\right\|_{L^{1}} & \leq \frac{2}{\Gamma\left(\frac{1-\alpha}{2}\right)} \int_{0}^{\infty} d t t^{\frac{1-\alpha}{2}} e^{-\frac{m^{2}}{2} t} \int_{0}^{1} d \theta\left(\frac{1}{2 e \frac{(1-\theta) t}{2}}\right)^{1 / 2} C_{\chi, A}\|\nabla \psi\|_{L^{\infty}}\|u\|_{L^{2}} \\
& \leq \frac{2}{\Gamma\left(\frac{1-\alpha}{2}\right)} \int_{0}^{\infty} d t t^{-\frac{\alpha}{2}} e^{-\frac{m^{2}}{2} t} \int_{0}^{1} \frac{d \theta}{(1-\theta)^{1 / 2}}(2 e)^{-1 / 2} C_{\chi, A}\|\nabla \psi\|_{L^{\infty}}\|u\|_{L^{2}},
\end{aligned}
$$

of which the last member yields (3.7) with (3.8).

Thus, taking (3.5) into account and putting together (3.6) and (3.7), we see the $L^{1}$ norm of the second term on the right-hand side of (3.3) is estimated as

$$
\begin{aligned}
& \left\|\chi\left[\psi,\left(H_{A, m}\right)^{-(1-\alpha)}\right] H_{A, m} u\right\|_{L^{1}} \\
& \leq\left\|I_{5} u\right\|_{L^{1}}+\left\|I_{6} u\right\|_{L^{1}} \\
& \leq \frac{\Gamma\left(\frac{2-\alpha}{2}\right)}{\Gamma\left(\frac{1-\alpha}{2}\right)}\left(\frac{2}{m^{2}}\right)^{\frac{2-\alpha}{2}}\left[2^{\frac{3}{2}}(2 e)^{-1 / 2}\|\chi\|_{L^{2}}\|\Delta \psi\|_{L^{2}}+4 \pi(2 e)^{-1} C_{\chi, A}\|\nabla \psi\|_{L^{\infty}}\right]\|u\|_{L^{2}} .
\end{aligned}
$$


Since the last member of (3.14) tends to zero as $\alpha \uparrow 1$, because $\Gamma(z) \uparrow \infty$ as $z \downarrow 0$ and hence $\frac{1}{\Gamma\left(\frac{1-\alpha}{2}\right)} \rightarrow 0$ as $\alpha \uparrow 1$, we see the left-hand side is uniformly bounded for $0<\alpha<1$, and convergent to zero as $\alpha \uparrow 1$. This shows the desired assertion of Lemma 3.2 .

Now we are in a position to prove Theorem 1.1.

Completion of Proof of Theorem 1.1.

As (1.2) and (1.3) are equivalent, we have only to show (1.3). The proof is divided into three parts, (i) the case where $m>0$ and $0<\alpha<1$, (ii) the case where $m>0$ and $\alpha=1$, (iii) the case where $m=0$ and $\alpha=1$.

(i) The case where $m>0$ and $0<\alpha<1$. We prove in two steps, treating first the step (i-I) for $u \in\left(C^{\infty} \cap L^{2}\right)\left(\mathbb{R}^{d}\right)$, and next the step (i-II) for general $u \in L^{2}$ with $\left(H_{A, m}\right)^{\alpha} u \in L_{\text {loc }}^{1}$

(i-I) For $u \in\left(C^{\infty} \cap L^{2}\right)\left(\mathbb{R}^{d}\right)(0<\alpha<1)$.

For a function $v(x) \in C^{\infty}\left(\mathbb{R}^{d}\right)$ and $\varepsilon>0$, put $v_{\varepsilon}(x)=\sqrt{|v(x)|^{2}+\varepsilon^{2}}$. Then note that $v_{\varepsilon}(x) \geq \varepsilon$, and, since $v_{\varepsilon}(x)^{2}=|v(x)|^{2}+\varepsilon^{2}$, we have

$$
-|v(x)||v(x+y)|+|v(x)|^{2} \geq-v_{\varepsilon}(x) v_{\varepsilon}(x+y)+v_{\varepsilon}(x)^{2} .
$$

Then we will show that $u_{\varepsilon}=\sqrt{|u|^{2}+\varepsilon^{2}}, \varepsilon>0$, satisfies that

$\operatorname{Re}\left[\overline{u(x)}\left(\left[\left(H_{A, m}\right)^{\alpha}-m^{\alpha}\right] u\right)(x)\right] \geq u_{\varepsilon}(x)\left(\left[\left(H_{0, m}\right)^{\alpha}-m^{\alpha}\right]\left(u_{\varepsilon}-\varepsilon\right)\right)(x), \quad$ pointwise a.e.,

which amounts to the same thing as

$$
\operatorname{Re}\left[\frac{\overline{u(x)}}{u_{\varepsilon}(x)}\left(\left[\left(H_{A, m}\right)^{\alpha}-m^{\alpha}\right] u\right)(x)\right] \geq\left(\left[\left(H_{0, m}\right)^{\alpha}-m^{\alpha}\right]\left(u_{\varepsilon}-\varepsilon\right)\right)(x),
$$

pointwise a.e., and thus in distributional sense. Here note that the function $u_{\varepsilon}-\varepsilon$ is nonnegative, $C^{\infty}$ and has the same compact support as $u$.

We show (3.16) or (3.17) first for $u \in C_{0}^{\infty}\left(\mathbb{R}^{d}\right)$ and then for $u \in\left(C^{\infty} \cap L^{2}\right)\left(\mathbb{R}^{d}\right)$. To do so, we employ analogous arguments as used in [I93, p.223, Lemma 2] for the case $\alpha=$ 1, i.e. for $H_{A, m}-m$. We will use the same notation $S$ as in Section 2 for the selfadjoint operator $(-i \nabla-A(x))^{2}+m^{2}$ in $L^{2}\left(\mathbb{R}^{d}\right)$, which may be considered as the magnetic nonrelativistic Schrödinger operator with mass $\frac{1}{2}$ with constant scalar potential $\mathrm{m}^{2}$. Then we have $H_{A, m}=S^{\frac{1}{2}}$. Since the domain of $H_{A, m}$ includes $C_{0}^{\infty}\left(\mathbb{R}^{d}\right)$ as a operator core, the operator $\left[H_{A, m}-m\right] u$ can be written as s- $\lim _{t \downarrow 0} t^{-1}\left(1-e^{-t\left[H_{A, m}-m\right]}\right) u$. It is known from the theory of fractional powers of a linear operator (e.g. see [Y78, IX, 11, pp.259-261]) that the semigroup $e^{-t\left[\left(H_{A, m}\right)^{\alpha}-m^{\alpha}\right]}$ with generator $\left(H_{A, m}\right)^{\alpha}=S^{\frac{\alpha}{2}}$ is obtained from the semigroup $e^{-t S}$ with generator $S$ as

$$
e^{-t\left[\left(H_{A, m}\right)^{\alpha}-m^{\alpha}\right]} u= \begin{cases}e^{m^{\alpha} t} \int_{0}^{\infty} f_{t, \frac{\alpha}{2}}(\lambda) e^{-\lambda S} u d \lambda, & t>0 \\ u, & t=0\end{cases}
$$

where for $t>0$ and $0<\alpha \leq 1, f_{t, \frac{\alpha}{2}}(\lambda)$ is a nonnegative function of exponential growth in $\lambda \in \mathbb{R}$ given by

$$
f_{t, \frac{\alpha}{2}}(\lambda)= \begin{cases}(2 \pi i)^{-1} \int_{\sigma-i \infty}^{\sigma+i \infty} e^{z \lambda-t z^{\frac{\alpha}{2}}} d z, & \lambda \geq 0 \\ 0, & \lambda<0\end{cases}
$$


with $\sigma>0$, where the branch of $z^{\frac{\alpha}{2}}$ is so taken that $\operatorname{Re} z^{\frac{\alpha}{2}}>0$ for $\operatorname{Re} z>0$. In passing, we note that equation (3.18) is valid even for $1<\alpha<2$, though we don not need this case in the present paper.

We continue our preceding arguments and recall that $\left|e^{-t S} u\right| \leq e^{-t\left[-\Delta+m^{2}\right]}|u|$ pointwise a.e., what is referred to in (2.17). It follows with (3.18), (3.19), that

$$
\begin{aligned}
\left|e^{-t\left[\left(H_{A, m}\right)^{\alpha}-m^{\alpha}\right]} u\right| & \leq e^{m^{\alpha} t} \int_{0}^{\infty} f_{t, \frac{\alpha}{2}}(\lambda)\left|e^{-\lambda S} u\right| d \lambda \\
& \leq e^{m^{\alpha} t} \int_{0}^{\infty} f_{t, \frac{\alpha}{2}}(\lambda) e^{-\lambda\left(-\Delta+m^{2}\right)}|u| d \lambda \\
& =e^{-t\left[\left(H_{0, m}\right)^{\alpha}-m^{\alpha}\right]}|u|,
\end{aligned}
$$

poitwise a.e. Hence for $t>0$

$$
\operatorname{Re}\left[\overline{u(x)}\left(\frac{1-e^{-t\left[\left(H_{A, m}\right)^{\alpha}-m^{\alpha}\right]}}{t} u\right)(x)\right] \geq|u(x)|\left(\frac{1-e^{-t\left[\left(H_{0, m}\right)^{\alpha}-m^{\alpha}\right]}}{t}|u|\right)(x),
$$

poitwise a.e. Now put $n^{m, \alpha}(t, y):=\frac{1}{t} k_{0}^{m, \alpha}(t, y)$, taking account of the relation (2.7) between the integral kernel $k_{0}^{m, \alpha}(t, y)$ of $e^{-t\left[\left(H_{0, m}\right)^{\alpha}-m^{\alpha}\right]}$ and the density (function) $n^{m, \alpha}(y)$ of the Lévy measure.

Then we see, by (2.6), that the right-hand side of (3.21) equal to

$$
\begin{aligned}
& |u(x)| \int_{|y|>0}[|u(x)|-|u(x+y)|] \frac{k_{0}^{m, \alpha}(t, y)}{t} d y \\
& =-\int_{|y|>0}\left[|u(x)||u(x+y)|-|u(x)|^{2}\right] n^{m, \alpha}(t, y) d y \\
& \geq-\int_{|y|>0}\left[u_{\varepsilon}(x) u_{\varepsilon}(x+y)-u_{\varepsilon}(x)^{2}\right] n^{m, \alpha}(t, y) d y \\
& =u_{\varepsilon}(x)\left[-\int_{|y|>0}\left[u_{\varepsilon}(x+y)-u_{\varepsilon}(x)-I_{\{|y|<1\}} y \cdot \nabla u_{\varepsilon}(x)\right] n^{m, \alpha}(t, y) d y\right],
\end{aligned}
$$

for every $\varepsilon>0$, where we used (3.15) and the $y$-rotational invariance of $k_{0}^{m, \alpha}(t, y)$ or $n^{m, \alpha}(t, y)$. Notice the integral $\left[-\int_{|y|>0} \cdots\right]$ of the last member is equal to that with $\left(u_{\varepsilon}-\varepsilon\right)$ in place of $u_{\varepsilon}$, i.e.

$$
\left(\frac{1-e^{-t\left[\left(H_{0, m}\right)^{\alpha}-m^{\alpha}\right]}}{t}\left(u_{\varepsilon}-\varepsilon\right)\right)(x) .
$$

Thus we have from (3.21)

$$
\operatorname{Re}\left[\overline{u(x)}\left(\frac{1-e^{-t\left[\left(H_{A, m}\right)^{\alpha}-m^{\alpha}\right]}}{t} u\right)(x)\right] \geq u_{\varepsilon}(x)\left(\frac{1-e^{-t\left[\left(H_{0, m}\right)^{\alpha}-m^{\alpha}\right]}}{t}\left(u_{\varepsilon}-\varepsilon\right)\right)(x) .
$$

Then letting $t \downarrow 0$ on both sides of (3.22), we obtain (3.16). Indeed, recalling the function $u_{\varepsilon}-\varepsilon$ has compact support, the right-hand side tends to that of (3.16). For the lefthand side, since $u$ is in the domain of $\left(H_{A, m}\right)^{\alpha}-m^{\alpha}$, we have $t^{-1}\left[1-e^{-t\left[\left(H_{A, m}\right)^{\alpha}-m^{\alpha}\right]}\right] u \rightarrow$ $\left[\left(H_{A, m}\right)^{\alpha}-m^{\alpha}\right] u$ in $L^{2}$, and pointwise a.e. by passing to a subsequence. This shows $(3.16) /(3.17)$ for $u \in C_{0}^{\infty}\left(\mathbb{R}^{d}\right)$.

Next we show $(3.16) /(3.17)$ when $u \in\left(C^{\infty} \cap L^{2}\right)\left(\mathbb{R}^{d}\right)$. Take a sequence $\left\{u_{n}\right\} \in$ $C_{0}^{\infty}\left(\mathbb{R}^{d}\right)$ such that $u_{n} \rightarrow u$ in $\left(C^{\infty} \cap L^{2}\right)\left(\mathbb{R}^{d}\right)$, i.e. in the topology of $C^{\infty}\left(\mathbb{R}^{d}\right)$ as well 
as in the norm of $L^{2}\left(\mathbb{R}^{d}\right)$, as $n \rightarrow \infty$. Then from the case $u \in C_{0}^{\infty}\left(\mathbb{R}^{d}\right)$ above, we have for all $\varepsilon>0$

$$
\operatorname{Re}\left[\frac{\overline{u_{n}(x)}}{u_{n, \varepsilon}(x)}\left(\left[\left(H_{A, m}\right)^{\alpha}-m^{\alpha}\right] u_{n}\right)(x)\right] \geq\left(\left[\left(H_{0, m}\right)^{\alpha}-m^{\alpha}\right]\left(u_{n, \varepsilon}-\varepsilon\right)\right)(x),
$$

pointwise, and hence for any $\psi \in C_{0}^{\infty}\left(\mathbb{R}^{d}\right)$ with $\psi(x) \geq 0$,

$$
\operatorname{Re}\left\langle\psi, \frac{\overline{u_{n}}}{u_{n, \varepsilon}}\left(\left[\left(H_{A, m}\right)^{\alpha}-m^{\alpha}\right] u_{n}\right)\right\rangle \geq\left\langle\psi,\left[\left(H_{0, m}\right)^{\alpha}-m^{\alpha}\right]\left(u_{n, \varepsilon}-\varepsilon\right)\right\rangle
$$

for all $\varepsilon>0$. Here the bilinear inner product $\langle\cdot, \cdot\rangle$ is an integral with respect to the Lebesgue measure $d x$, and also considered as the bilinear inner product between the dual pair of the test functions and the distributions: $\left\langle C_{0}^{\infty}\left(\mathbb{R}^{d}\right), \mathcal{D}^{\prime}\left(\mathbb{R}^{d}\right)\right\rangle$. Therefore

$$
\operatorname{Re}\left\langle\left[\left(H_{A, m}\right)^{\alpha}-m^{\alpha}\right]\left(\frac{\overline{u_{n}}}{u_{n, \varepsilon}} \psi\right), u_{n}\right\rangle \geq\left\langle\left[\left(H_{0, m}\right)^{\alpha}-m^{\alpha}\right] \psi, u_{n, \varepsilon}-\varepsilon\right\rangle .
$$

Since we have that $u_{n} \rightarrow u$ and $u_{n, \varepsilon} \rightarrow u_{\varepsilon}$ in $\left(C^{\infty} \cap L^{2}\right)\left(\mathbb{R}^{d}\right)$ as $n \rightarrow \infty$, we have that $\left(\frac{\overline{u_{n}}}{u_{n, \varepsilon}}\right) \psi \rightarrow\left(\frac{\bar{u}}{u_{\varepsilon}}\right) \psi$. It follows by Lemma 2.2 that

$$
\left[\left(H_{A, m}\right)^{\alpha}-m^{\alpha}\right]\left(\frac{\overline{u_{n}}}{u_{n, \varepsilon}}\right) \psi \rightarrow\left(\left[H_{A, m}\right)^{\alpha}-m^{\alpha}\right]\left(\frac{\bar{u}}{u_{\varepsilon}}\right) \psi
$$

in $L^{2}$ as $n \rightarrow \infty$, so that

$$
\operatorname{Re}\left\langle\left[\left(H_{A, m}\right)^{\alpha}-m^{\alpha}\right]\left(\frac{\bar{u}}{u_{\varepsilon}} \psi\right), u\right\rangle \geq\left\langle\left[\left(H_{0, m}\right)^{\alpha}-m^{\alpha}\right] \psi,\left(u_{\varepsilon}-\varepsilon\right)\right\rangle .
$$

Thus we obtain

$$
\operatorname{Re}\left[\frac{\overline{u(x)}}{u_{\varepsilon}(x)}\left(\left[\left(H_{A, m}\right)^{\alpha}-m^{\alpha}\right] u\right)(x)\right] \geq\left(\left[\left(H_{0, m}\right)^{\alpha}-m^{\alpha}\right]\left(u_{\varepsilon}-\varepsilon\right)\right)(x),
$$

pointwise a.e., and thus in distributional sense, and hence (3.17) follows for $u \in\left(C^{\infty} \cap\right.$ $\left.L^{2}\right)\left(\mathbb{R}^{d}\right)$.

(i-II) For general $u \in L^{2}\left(\mathbb{R}^{d}\right)$ with $\left(H_{A, m}\right)^{\alpha} u \in L_{\text {loc }}^{1}\left(\mathbb{R}^{d}\right)(0<\alpha<1)$.

Put $u^{\delta}=\rho_{\delta} * u$. Then $u^{\delta} \in C^{\infty} \cap L^{2}$, so by (3.23) in step (i-I) above

$$
\operatorname{Re}\left[\frac{\overline{u^{\delta}}}{\left(u^{\delta}\right)_{\varepsilon}}\left(\left[\left(H_{A, m}\right)^{\alpha}-m^{\alpha}\right] u^{\delta}\right)\right] \geq\left[\left(H_{0, m}\right)^{\alpha}-m^{\alpha}\right]\left(\left(u^{\delta}\right)_{\varepsilon}-\varepsilon\right),
$$

pointwise a.e., and also in distributional sense, for all $\varepsilon>0$ and all $\delta>0$.

We first, for fixed $\varepsilon>0$, let $\delta \downarrow 0$, and next $\varepsilon \downarrow 0$. In fact, if $\delta \downarrow 0$, then $u^{\delta} \rightarrow u$ in $L^{2}$ as well as a.e. by passing to a subsequence of $\left\{u^{\delta}\right\}$. Hence $\overline{u^{\delta}} /\left(u^{\delta}\right)_{\varepsilon} \rightarrow \bar{u} / u_{\varepsilon}$ a.e. and by Lemma 3.1, $\left(H_{A, m}\right)^{\alpha} u^{\delta} \rightarrow\left(H_{A, m}\right)^{\alpha} u$ locally in $L^{1}$, and therefore also a.e. by passing to a subsequence. Since $\left|\frac{\overline{u^{\delta}}}{\left(u^{\delta}\right)_{\varepsilon}}\right| \leq 1$, it follows by the Lebesque dominated convergence theorem that on the left-hand side of (3.24),

$$
\frac{\overline{u^{\delta}}}{\left(u^{\delta}\right)_{\varepsilon}}\left[\left(H_{A, m}\right)^{\alpha}-m^{\alpha}\right] u^{\delta} \rightarrow \frac{\bar{u}}{u_{\varepsilon}}\left[\left(H_{A, m}\right)^{\alpha}-m^{\alpha}\right] u
$$


locally in $L^{1}$ as $\delta \downarrow 0$. On the other hand, for the right-hand side, since

$$
\left|\left(\left(u^{\delta}\right)_{\varepsilon}-\varepsilon\right)-\left(u_{\varepsilon}-\varepsilon\right)\right| \leq\left|\left(u^{\delta}\right)_{\varepsilon}-u_{\varepsilon}\right| \leq|| u^{\delta}|-| u|| \leq\left|u^{\delta}-u\right|,
$$

we have $\left(H_{0, m}\right)^{\alpha}\left(\left(u^{\delta}\right)_{\varepsilon}-\varepsilon\right) \rightarrow\left(H_{0, m}\right)^{\alpha}\left(u_{\varepsilon}-\varepsilon\right)$ in $\mathcal{D}^{\prime}$ (in distributional sense). This shows that (3.23) holds for $u \in L^{2}\left(\mathbb{R}^{d}\right)$ with $\left(H_{A, m}\right)^{\alpha} u \in L_{\text {loc }}^{1}\left(\mathbb{R}^{d}\right)$. Next let $\varepsilon \downarrow$ 0 . Then $\bar{u} / u_{\varepsilon} \rightarrow \operatorname{sgn} u$ a.e. with $\left|\bar{u} / u_{\varepsilon}\right| \leq 1$, so that the left-hand side of (3.23) converges to $\operatorname{Re}\left((\operatorname{sgn} u)\left[H_{A, m}-m\right] u\right)$ a.e., while the right-hand side of (3.23) converges to $\left[\left(H_{0, m}\right)^{\alpha}-m^{\alpha}\right]|u|$ in $\mathcal{D}^{\prime}$. Thus we get (3.1), showing the desired inequality for $0<\alpha<1$.

(ii) The case where $m>0$ and $\alpha=1$.

Once the inequality (3.1) is established for $0<\alpha<1$, we let $\alpha \uparrow 1$, with $u \in$ $L^{2}\left(\mathbb{R}^{d}\right)$ with $H_{A, m} u \in L_{\text {loc }}^{1}\left(\mathbb{R}^{d}\right)$. Then, as $\alpha \uparrow 1$, by Lemma 3.2 we have $\left(H_{A, m}\right)^{\alpha} u \rightarrow$ $H_{A, m} u$ in $L_{\text {loc }}^{1}$ and also trivially $m^{\alpha} \rightarrow m$. The left-hand side of (3.1) converges to $\operatorname{Re}\left((\operatorname{sgn} u)\left[H_{A, m}-m\right] u\right)$ in $L_{\text {loc }}^{1}$, while the right-hand side converges to $\left[H_{0, m}-m\right]|u|$ in distributional sense, so that we have shown the desired inequality (1.3).

(iii) The case where $m=0$ and $\alpha=1$. This follows from the case (ii) for $m>0$, i.e. by letting $m \downarrow 0$ in the equality (1.3) with $m>0$. To see this, let $u \in L^{2}\left(\mathbb{R}^{d}\right)$ with $H_{A, 0} u \in L_{\text {loc }}^{1}\left(\mathbb{R}^{d}\right)$. Then, noting that $H_{A, 0}=|-i \nabla-A|$, we see by the argument done around (2.1) that the domains of the operators $H_{A, m}$ and $H_{A, 0}$ coincide. We also see that $H_{A, 0} u \in L_{\text {loc }}^{1}\left(\mathbb{R}^{d}\right)$ with $u \in L^{2}\left(\mathbb{R}^{d}\right)$ implies $H_{A, m} u \in L_{\text {loc }}^{1}\left(\mathbb{R}^{d}\right)$. In fact, we can show the following fact.

Lemma 3.3. Let $u \in L^{2}\left(\mathbb{R}^{d}\right)$. Then $H_{A, m} u \in L_{\mathrm{loc}}^{1}\left(\mathbb{R}^{d}\right)$ if and only if $H_{A, 0} u \in L_{\mathrm{loc}}^{1}\left(\mathbb{R}^{d}\right)$. In fact, for $\psi \in C_{0}^{\infty}\left(\mathbb{R}^{d}\right)$ it holds that

$$
\left|\left\|\psi H_{A, m} u\right\|_{L^{1}}-\left\|\psi H_{A, 0} u\right\|_{L^{1}}\right| \leq C(d) m^{2}\|\psi\|_{L^{\frac{2 d}{d+2}}}\|u\|_{L^{2}}
$$

with a constant $C(d)$ depending only on $d$.

Proof. We have for $\phi \in C_{0}^{\infty}\left(\mathbb{R}^{d}\right)$

$$
\begin{aligned}
H_{A, m} \phi-H_{A, 0} \phi & =\left((-\nabla-A)^{2}+m^{2}\right)^{1 / 2} \phi-|-i \nabla-A| \phi \\
& =\left[\left((-\nabla-A)^{2}+\theta m^{2}\right)^{1 / 2} \phi\right]_{\theta=0}^{\theta=1}=\int_{0}^{1} \frac{d}{d \theta}\left[\left((-\nabla-A)^{2}+\theta m^{2}\right)^{1 / 2} \phi\right] d \theta \\
& =\frac{m^{2}}{2} \int_{0}^{1}\left((-\nabla-A)^{2}+\theta m^{2}\right)^{-1 / 2} \phi d \theta
\end{aligned}
$$

Multiply (3.26) by $\psi \in C_{0}^{\infty}\left(\mathbb{R}^{d}\right)$ with $\psi(x) \geq 0$, and integrate the absolute value in $x$, then we have

$$
\begin{aligned}
\left\|\psi H_{A, m} \phi-\psi H_{A, 0} \phi\right\|_{L^{1}} & \leq \frac{m^{2}}{2} \int_{0}^{1}\left\|\psi\left((-\nabla-A)^{2}+\theta m^{2}\right)^{-1 / 2} \phi\right\|_{L^{1}} d \theta \\
& \leq \frac{m^{2}}{2} \int_{0}^{1}\left\|\psi\left(-\Delta+\theta m^{2}\right)^{-1 / 2}|\phi|\right\|_{L^{1}} d \theta \\
& =\frac{m^{2}}{2} \int_{0}^{1} \int_{\mathbb{R}^{d}}\left[\psi\left(-\Delta+\theta m^{2}\right)^{-1 / 2}|\phi|\right](x) d x d \theta
\end{aligned}
$$


where the second inequality is due to Lemma 2.1 (i) with $\beta=\frac{1}{2}$ and $p=1$. Note also that the operator $\left(-\Delta+m^{2}\right)^{-1 / 2}$ in $(3.27)$ has the following positive integral kernel:

$$
\left(-\Delta+m^{2}\right)^{-1 / 2}(x)=\frac{2 m^{d-1}}{(2 \pi)^{\frac{d+1}{2}}} \frac{K_{(d-1) / 2}(m|x|)}{(m|x|)^{(d-1) / 2}}, \quad m>0,
$$

with $K_{\nu}(\tau)$ the modified Bessel function of the third kind of order $\nu$, which was also referred to around $(2.8) /(2.9)$. In fact, using the expression (2.9) for the integral kernel of $e^{-t H_{0, m}}=e^{-\left[-\Delta+m^{2}\right]^{-1 / 2}}$ and integrating it in $t$ on $(0, \infty)$, we have

$$
\begin{aligned}
\left(-\Delta+m^{2}\right)^{-1 / 2}(x) & =\int_{0}^{\infty} k_{0}^{m, 1}(t, x) \cdot e^{-m t} d t \\
& =\int_{0}^{\infty} 2\left(\frac{m}{2 \pi}\right)^{\frac{d+1}{2}} \frac{t K_{(d+1) / 2}\left(m\left(x^{2}+t^{2}\right)^{1 / 2}\right)}{\left(x^{2}+t^{2}\right)^{(d+1) / 4}} d t
\end{aligned}
$$

Change the variables $\tau=m\left(x^{2}+t^{2}\right)^{1 / 2}$, so that $2 t d t=\frac{2 \tau}{m^{2}} d \tau$, and use $\frac{d}{\tau d \tau} \frac{K_{\nu}(\tau)}{\tau^{\nu}}=$ $-\frac{K_{\nu+1}(\tau)}{\tau^{\nu+1}}$, then we see the last member above be equal to

$$
\begin{aligned}
\int_{m|x|}^{\infty} \frac{m^{\frac{d+1}{2}}}{(2 \pi)^{\frac{d+1}{2}}} \frac{K_{(d+1) / 2}(\tau)}{(\tau / m)^{\frac{d+1}{2}}} \frac{2 \tau}{m^{2}} d \tau & =-\frac{1}{(2 \pi)^{\frac{d+1}{2}}} \int_{m|x|}^{\infty} m^{d+1} \frac{d}{\tau d \tau}\left[\frac{K_{(d-1) / 2}(\tau)}{\tau^{\frac{d-1}{2}}}\right] \frac{2 \tau}{m^{2}} d \tau \\
& =\frac{2 m^{d-1}}{(2 \pi)^{\frac{d+1}{2}}} \frac{K_{(d-1) / 2}(m|x|)}{(m|x|)^{\frac{d-1}{2}}}
\end{aligned}
$$

which yields (3.28).

Since it holds that $0<K_{\nu}(\tau) \leq C\left[\tau^{-\nu} \vee \tau^{-1 / 2}\right] e^{-\tau}, \tau>0$ with a constant $C>0$ when $\nu>0$, we obtain

$$
\frac{K_{(d-1) / 2}\left(\theta^{1 / 2} m|x|\right)}{\left(\theta^{1 / 2} m|x|\right)^{\frac{d-1}{2}}} \leq C \frac{1}{\left(\theta^{1 / 2} m|x|\right)^{(d-1)}} .
$$

Then we see from (3.27) by the Hardy-Littlewood-Sobolev inequality (e.g. [LLos01, Chap.4, Sect. 4.3]), noting $p=\frac{2 d}{d+2}$ satisfies the relation $\frac{1}{p}+\frac{d-1}{d}+\frac{1}{2}=2$,

$$
\begin{aligned}
& \left\|\psi H_{A, m} \phi-\psi H_{A, 0} \phi\right\|_{L^{1}} \\
& \leq \frac{m^{d+1}}{(2 \pi)^{\frac{d+1}{2}}} \int_{0}^{1} d \theta \theta^{(d+1) / 2} \iint_{\mathbb{R}^{d} \times \mathbb{R}^{d}} \psi(x) \frac{K_{(d-1) / 2}\left(\theta^{1 / 2} m|x-y|\right)}{\left(\theta^{1 / 2} m|x-y|\right)^{\frac{d-1}{2}}}|\phi(y)| d x d y \\
& \leq \frac{C(d)}{2} \frac{m^{d+1}}{(2 \pi)^{\frac{d+1}{2}}} \int_{0}^{1} d \theta \theta m^{-(d-1)}\|\psi\|_{L^{\frac{2 d}{d+2}}}\|\phi\|_{L^{2}} \\
& =C(d) \frac{m^{2}}{(2 \pi)^{\frac{d+1}{2}}}\|\psi\|_{L^{\frac{2 d}{d+2}}}\|\phi\|_{L^{2}}
\end{aligned}
$$

with a constant $C(d)>0$ depending on $d$.

Now, to show the desired inequality (3.25), let $u \in L^{2}\left(\mathbb{R}^{d}\right)$ and assume that either $H_{A, m} u$ or $H_{A, 0} u$ in $L_{\text {loc }}^{1}\left(\mathbb{R}^{d}\right)$, consider, for instance, the latter $H_{A, 0} u \in L_{\text {loc }}^{1}\left(\mathbb{R}^{d}\right)$. There exists a sequence $\left\{\phi_{n}\right\}_{n=1}^{\infty}$ in $C_{0}^{\infty}\left(\mathbb{R}^{d}\right)$ convergent to $u$ in $L^{2}$ as $n \rightarrow \infty$. We see by 
(3.29) that $\left\{\left(\psi H_{A, m}-\psi H_{A, 0}\right) \phi_{n}\right\}_{n=1}^{\infty}$ is a Cauchy sequence in $L^{1}$, so that there exists $v \in L^{1}\left(\mathbb{R}^{d}\right)$ to which it converges in $L^{1}$ :

$$
\left(\psi H_{A, m}-\psi H_{A, 0}\right) \phi_{n} \rightarrow v, \quad n \rightarrow \infty .
$$

Since $\psi D\left[H_{A, 0}\right] \subseteq D\left[H_{A, 0}\right]$, we see $\left\{\psi H_{A, 0} \phi_{n}\right\}$ converge to $\psi H_{A, 0} u \in L^{1}\left(\mathbb{R}^{d}\right)$ in the weak topology defined by the dual pairing $\left\langle L^{1}\left(\mathbb{R}^{d}\right), D\left[H_{A, 0}\right]\right\rangle$. So $\left\{\psi H_{A, m} \phi_{n}\right\}$ becomes a Cauchy sequence also in this weak topology $\sigma\left(L^{1}\left(\mathbb{R}^{d}\right), D\left[H_{A, 0}\right]\right)$, converging to $v-\psi H_{A, 0} u$, which also belongs to $L^{1}\left(\mathbb{R}^{d}\right)$. Therefore the existing limit of $\left\{\psi H_{A, m} \phi_{n}\right\}$ should be written as $\psi H_{A, m} u$ to satisfy

$$
v=\psi H_{A, m} u+\psi H_{A, 0} u .
$$

Thus we have seen (3.29) implies

$$
\left\|\psi H_{A, m} u-\psi H_{A, 0} u\right\|_{L^{1}} \leq C(d) m^{2}\|\psi\|_{L^{\frac{2 d}{d+2}}}\|u\|_{L^{2}}
$$

Hence we have by the triangle inequality ||$a|-| b|| \leq|a-b|$ we have (3.25). This shows (3.25) for the general $u$, ending the proof of Lemma 3.3.

Finally, we come back to the proof of Theorem 1.1 to continue the case (iii) The case where $m=0$ and $\alpha=1$. We show that, as $m \downarrow 0$, the left-hand side and the right-hand side of (1.3) with $m>0$ converge to those with $m=0$.

As for the left-hand side, the sequence $\left\{\left\|\left[H_{A, m}-m\right] u\right\|_{L^{2}}^{2}\right\}$ of quadratic forms is increasing as $m$ decreases and converges to $\left\|H_{A, 0} u\right\|_{L^{2}}^{2}$ as $m \downarrow 0$, because

$$
\left[H_{A, m}-m\right]=\frac{(-i \nabla-A)^{2}}{H_{A, m}+m} \leq \frac{(-i \nabla-A)^{2}}{H_{A}^{m^{\prime}}+m^{\prime}}=\left[H_{A}^{m^{\prime}}-m^{\prime}\right] \leq H_{A, 0}=|-i \nabla-A|
$$

for $m \geq m^{\prime}>0$. This shows the convergence of the left-hand side of (1.3). As for the right-hand side, it is easy to see that, as $m \downarrow 0, H_{0, m}|u| \equiv\left(-\Delta+m^{2}\right)^{\frac{1}{2}}|u|$ converges to $H_{0}^{0}|u| \equiv(-\Delta)^{\frac{1}{2}}|u|$ in the distribution sense, because one can show that, for any $\psi \in C_{0}^{\infty}\left(\mathbb{R}^{n}\right),\left\{H_{0, m} \psi\right\}$ converges to $H_{0}^{0} \psi$ as $m \downarrow 0$, by using their integral operator representation formula (2.6) with $\alpha=1$; in fact, it is due to the convergence of the Lévy measure $n^{m, 1}(d y)$ to the Lévy measure $n^{0,1}(d y)$ on $\mathbb{R}^{d} \backslash\{0\}$, which amounts to the same thing as, observing (2.9), the convergence of density $n^{m, 1}(y)$ to density $n^{0,1}(y)$. This shows the case $m=0$, completing the proof of Theorem 1.1.

Remark. From the proof of Theorem 1.1 above, in particular, the step (i-II), which relies on Lemma 3.1, we see Theorem 1.1 (Kato's inequality) also hold for $\left(H_{A, m}\right)^{\alpha},\left(H_{0, m}\right)^{\alpha}$ in place of $H_{A, m}, H_{0, m}$ with $0<\alpha<1$, that is, (3.1) hold for $0<\alpha<1$ if $u \in L^{2}\left(\mathbb{R}^{d}\right)$ with $\left(H_{A, m}\right)^{\alpha} u \in\left[L_{\text {loc }}^{1}\left(\mathbb{R}^{d}\right)\right]^{d}$. As a result, Theorem 1.2 (Diamagnetic inequality) also holds for $\left(H_{A, m}\right)^{\alpha},\left(H_{0, m}\right)^{\alpha}$.

\subsection{Proof of Theorem 1.2}

This has already been implicitly shown in the proof of Theorem 1.1. In fact, by the same argument used to get (3.20) from (3.18), (3.19), even for all $0<\alpha \leq 1$, we have 
for $f, g \in C_{0}^{\infty}\left(\mathbb{R}^{d}\right)$

$$
\begin{aligned}
\left|\left(f, e^{-t\left[\left(H_{A, m}\right)^{\alpha}-m^{\alpha}\right]} g\right)\right| & \leq e^{m^{\alpha} t} \int_{0}^{\infty} f_{t, \frac{\alpha}{2}}(\lambda)\left|\left(f, e^{-\lambda S} g\right)\right| d \lambda \\
& \leq e^{m^{\alpha} t} \int_{0}^{\infty} f_{t, \frac{\alpha}{2}}(\lambda)\left(|f|,\left|e^{-\lambda S} g\right|\right) d \lambda \\
& \leq e^{m^{\alpha} t} \int_{0}^{\infty} f_{t, \frac{\alpha}{2}}(\lambda)\left(|f|, e^{-\lambda\left(-\Delta+m^{2}\right)}|g|\right) d \lambda \\
& =\left(|f|, e^{-t\left[\left(H_{0, m}\right)^{\alpha}-m^{\alpha}\right]}|g|\right) .
\end{aligned}
$$

Then this is of course also valid for $f, g \in L^{2}\left(\mathbb{R}^{d}\right)$.

\section{Concluding Remarks}

In the literature there are three kinds of relativistic Schrödinger operators for a spinless particle of mass $m \geq 0$ corresponding to the classical relativistic Hamiltonian symbol $\sqrt{(\xi-A(x))^{2}+m^{2}}$ with magnetic vector potential $A(x)$, dependent on how to quantize this symbol. One of them is of course the one $H_{A, m}$ in (1.1) treated in this paper, and the other two are defined as pseudo-differential operators, differing from $H_{A, m}$ defined as an operator-theoretical square root. In [I12, I13], their common and different properties were discussed mainly in connection with the corresponding path integral representations for their semigroups.

The other two relativistic Schrödinger operators are defined by oscillatory integrals, for $f \in C_{0}^{\infty}\left(\mathbb{R}^{d}\right)$, as

$$
\begin{aligned}
\left(H_{A, m}^{(1)} f\right)(x) & :=\frac{1}{(2 \pi)^{d}} \iint_{\mathbb{R}^{d} \times \mathbb{R}^{d}} e^{i(x-y) \cdot \xi} \sqrt{\left(\xi-A\left(\frac{x+y}{2}\right)\right)^{2}+m^{2}} f(y) d y d \xi \\
& =\frac{1}{(2 \pi)^{d}} \iint_{\mathbb{R}^{d} \times \mathbb{R}^{d}} e^{i(x-y) \cdot\left(\xi+A\left(\frac{x+y}{2}\right)\right)} \sqrt{\xi^{2}+m^{2}} f(y) d y d \xi ; \\
\left(H_{A, m}^{(2)} f\right)(x): & =\frac{1}{(2 \pi)^{d}} \iint_{\mathbb{R}^{d} \times \mathbb{R}^{d}} e^{i(x-y) \cdot \xi} \sqrt{\left(\xi-\int_{0}^{1} A((1-\theta) x+\theta y) d \theta\right)^{2}+m^{2}} f(y) d y d \xi \\
& =\frac{1}{(2 \pi)^{d}} \iint_{\mathbb{R}^{d} \times \mathbb{R}^{d}} e^{i(x-y) \cdot\left(\xi+\int_{0}^{1} A((1-\theta) x+\theta y) d \theta\right)} \sqrt{\xi^{2}+m^{2}} f(y) d y d \xi .
\end{aligned}
$$

Equality (4.1) is a Weyl pseudo-differential operator with mid-point prescription given in [ITa86] (also [I89], [NaU90]) and (4.2) a modification of (4.1) given in [IfMP07]. Note that these two $H_{A, m}^{(1)}$ and $H_{A, m}^{(2)}$ are denoted in [I12, I13] by slightly different notations $H_{A}^{(1)}$ and $H_{A}^{(2)}$, respectively, while our $H_{A, m}$ in (1.1) by $H_{A}^{(3)}$.

What in this section we should like to call attention to is that Kato's inequality of distributional form was missing for $H_{A, m}^{(3)}$ or our $H_{A, m}$ in (1.1), although there already exist for the other two $H_{A, m}^{(1)}$ in (4.1), $H_{A, m}^{(2)}$ in (4.2), indeed it was shown for $H_{A, m}^{(1)}$ in [I89, ITs92] under some suitable conditions on $A(x)$ (which differ from $A \in L_{\text {loc }}^{2}$ ), and to be shown in the same way for $H_{A, m}^{(2)}$ (cf. [I13]). Therefore, at least the case of Theorem 1.1 with $A=0$ turns out to have already been known.

Let us briefly mention here some known facts for $H_{A, m}^{(1)}, H_{A, m}^{(2)}$ and $H_{A, m}^{(3)}$. 
$1^{\circ}$. With reasonable conditions on $A(x)$, they all define selfadjoint operators in $L^{2}\left(\mathbb{R}^{d}\right)$, which are bounded below. For instance, they become selfadjoint operators defined as quadratic forms, for $H_{A, m}^{(1)}$ and $H_{A, m}^{(2)}$, when $A \in L_{\mathrm{loc}}^{1+\delta}\left(\mathbb{R}^{d} ; \mathbb{R}^{d}\right)$ for some $\delta>0$ (cf. [I89, I13], [IfMP07]), while for $H_{A, m}^{(3)}$, when $A \in L_{\text {loc }}^{2}\left(\mathbb{R}^{d} ; \mathbb{R}^{d}\right)$ (e.g. [CFKiSi87, pp.8-10] or [I13]).

Furthermore, they are bounded below by the same lower bound, in particular,

$$
H_{A, m}^{(j)} \geq m, \quad j=1,2,3 .
$$

$2^{\circ} . H_{A, m}^{(2)}$ and $H_{A, m}^{(3)}$ are covariant under gauge transformation, i.e. for every $\varphi \in$ $\mathcal{S}\left(\mathbb{R}^{d}\right)$ it holds that $H_{A+\nabla \varphi}^{(j)}=e^{i \varphi} H_{A, m}^{(j)} e^{-i \varphi}, j=2,3$. However, $H_{A, m}^{(1)}$ is not.

$3^{\circ}$. All these three operators are different in general, but coincide, if $A(x)$ is linear in $x$, i.e. if $A(x)=\dot{A} \cdot x$ with $\dot{A}: d \times d$ real symmetric constant matrix, then $H_{A, m}^{(1)}=H_{A, m}^{(2)}=H_{A, m}^{(3)}$. So, this holds for uniform magnetic fields with $d=3$.

\section{Appendix A}

Our aim is to derive the following expressions for integral kernel $k_{0}^{m, \alpha}(t, x)$ of semigroup $e^{-t\left[\left(H_{0, m}\right)^{\alpha}-m^{\alpha}\right]}$ and density (function) $n^{m, \alpha}$ of Lévy measure $n^{m, \alpha}(d y)$ for $0<\alpha \leq 1$, which are mentioned around formulas $(2.7),(2.8) /(2.9)$ :

$$
\begin{aligned}
k_{0}^{m, \alpha}(t, x)= & \frac{e^{m^{\alpha} t}}{\pi(2 \pi)^{\frac{d}{2}}|x|^{\frac{d}{2}-1}} \int_{0}^{\infty} e^{-t r^{\frac{\alpha}{2}} \cos \frac{\alpha}{2} \pi} \sin \left(t r^{\frac{\alpha}{2}} \sin \frac{\alpha}{2} \pi\right)\left(m^{2}+r\right)^{\frac{1}{2}\left(\frac{d}{2}-1\right)} \\
\times K_{\frac{d}{2}-1}\left(\left(m^{2}+r\right)^{\frac{1}{2}}|x|\right) d r & \\
n^{m, \alpha}= & \frac{2^{1+\frac{\alpha}{2}} \sin \left(\frac{\alpha}{2} \pi\right)(2 \pi)^{\frac{\alpha}{2}} \Gamma\left(\frac{\alpha}{2}+1\right)}{\pi}\left(\frac{m}{2 \pi}\right)^{\frac{d+\alpha}{2}} \frac{K_{\frac{d+\alpha}{2}}(m|x|)}{|x|^{\frac{d+\alpha}{2}}} .
\end{aligned}
$$

Equality (A.2) is essentially the same as $\nu^{m}$ in [ByMaRy09, (2.7), p.4877], which is established for the heat semigroup $e^{-t\left[\left(-\Delta+m^{\alpha / 2}\right)^{\alpha / 2}-m\right]}$ instead of our $e^{-t\left[\left(H_{0, m}\right)^{\alpha}-m^{\alpha}\right]}$. Indeed, putting in (A.2) $m=m^{\prime \frac{1}{\alpha}}$ to rewrite it with Euler's reflection formula $\Gamma(z) \Gamma(1-$ $z)=\frac{\pi}{\sin (\pi z)}$ yields eq. (2.7) in this reference with $m$ replaced by $m^{\prime}$.

To show (A.1) and (A.2), we use another formula (3.18)/(3.19) to express the semigroup $e^{-t\left(H_{0, m}\right)^{\alpha}} \equiv e^{-t\left(-\Delta+m^{2}\right)^{\frac{\alpha}{2}}}(0<\alpha \leq 1)$ for the fractional power:

$$
\left(e^{-t\left(H_{0, m}\right)^{\alpha}} u\right)(x)=\int_{\mathbb{R}^{d}}\left(\int_{0}^{\infty} f_{t, \frac{\alpha}{2}}(s) e^{-s\left(-\Delta+m^{2}\right)} d s u\right)(y) d y,
$$

where $e^{-t\left(-\Delta+m^{2}\right)}$ is the heat semigroup multiplied by $e^{-m^{2} t}$ :

$$
\left(e^{-t\left(-\Delta+m^{2}\right)} u\right)(x)=\frac{1}{(4 \pi t)^{d / 2}} \int_{\mathbb{R}^{d}} e^{-m^{2} t-\frac{(x-y)^{2}}{4 t}} u(y) d y
$$

The $f_{t, \frac{\alpha}{2}}(s)$ in (3.19) is rewritten as

$$
f_{t, \frac{\alpha}{2}}(s)=\frac{1}{\pi} \int_{0}^{\infty} e^{s r \cos \theta-t r^{\alpha} \cos \frac{\alpha}{2} \theta} \sin \left(s r \sin \theta-t r^{\frac{\alpha}{2}} \sin \frac{\alpha}{2} \theta+\theta\right) d r \quad(t>0, s \geq 0),
$$


where the integration path is deformed to the union of two paths $r e^{-i \theta}(-\infty<-r<0)$ and $r e^{i \theta}(0<r<\infty)$, where $\frac{\pi}{2} \leq \theta \leq \pi$ (see [Y78, IX, 11, pp.259-263]).

Then we take $\theta=\pi$ to have

$$
\begin{gathered}
\left(e^{-t\left(H_{0, m}\right)^{\alpha}}\right)(x) \\
=\int_{0}^{\infty} d s \frac{1}{\pi} \frac{1}{(4 \pi s)^{\frac{d}{2}}} e^{-m^{2} s-\frac{x^{2}}{4 s}} \int_{0}^{\infty} e^{-s r-t r^{\frac{\alpha}{2}} \cos \frac{\alpha}{2} \pi} \sin \left(t r^{\frac{\alpha}{2}} \sin \frac{\alpha}{2} \pi\right) d r \\
=\frac{1}{\pi(4 \pi)^{\frac{d}{2}}} \int_{0}^{\infty} d r e^{-t r^{\frac{\alpha}{2}} \cos \frac{\alpha}{2} \pi} \sin \left(t r^{\frac{\alpha}{2}} \sin \frac{\alpha}{2} \pi\right)\left(m^{2}+r\right)^{\frac{d}{2}-1} \int_{0}^{\infty} \frac{e^{-s-\frac{\left(m^{2}+r\right) x^{2}}{4 s}}}{s^{\frac{d}{2}}} d s \\
=\frac{1}{\pi(2 \pi)^{\frac{d}{2}}|x|^{\frac{d}{2}-1}} \int_{0}^{\infty} e^{-t r^{\frac{\alpha}{2}} \cos \frac{\alpha}{2} \pi} \sin \left(t r^{\frac{\alpha}{2}} \sin \frac{\alpha}{2} \pi\right)\left(m^{2}+r\right)^{\frac{1}{2}\left(\frac{d}{2}-1\right)} \\
\times K_{\frac{d}{2}-1}\left(\left(m^{2}+r\right)^{\frac{1}{2}}|x|\right) d r,
\end{gathered}
$$

where we have used the representation formula of the modified Bessel function of the third kind, $K_{\nu}(z)$ [GrR94, sect. 8.432. 6, p.969]:

$$
K_{\nu}(z)=\frac{1}{2}\left(\frac{z}{2}\right)^{\nu} \int_{0}^{\infty} e^{-t-\frac{z^{2}}{4 t}} t^{-\nu-1} d t, \quad \nu>-\frac{1}{2}, z>0 .
$$

It follows that the integral kernel $k_{0}^{m, \alpha}(t, x)$ of the semigroup $e^{-t\left[\left(H_{0, m}\right)^{\alpha}-m^{\alpha}\right]}$ turns out

$$
\begin{aligned}
& k_{0}^{m, \alpha}(t, x):= e^{-t\left[\left(H_{0, m}\right)^{\alpha}-m^{\alpha}\right]}(x) \\
&=\frac{e^{m^{\alpha} t}}{\pi(2 \pi)^{\frac{d}{2}}|x|^{\frac{d}{2}-1}} \int_{0}^{\infty} e^{-t r^{\frac{\alpha}{2}} \cos \frac{\alpha}{2} \pi} \sin \left(t r^{\frac{\alpha}{2}} \sin \frac{\alpha}{2} \pi\right)\left(m^{2}+r\right)^{\frac{1}{2}\left(\frac{d}{2}-1\right)} \\
& \quad \times K_{\frac{d}{2}-1}\left(\left(m^{2}+r\right)^{\frac{1}{2}}|x|\right) d r .
\end{aligned}
$$

This shows (A.1).

Next, we have

$$
\begin{aligned}
& \frac{d}{d t} k_{0}^{m, \alpha}(t, x) \\
= & \frac{1}{\pi(2 \pi)^{\frac{d}{2}}|x|^{\frac{d-1}{2}}} \int_{0}^{\infty} d r \frac{d}{d t}\left[e^{t\left(m^{\alpha}-r^{\frac{\alpha}{2}} \cos \frac{\alpha}{2} \pi\right)} \sin \left(t r^{\frac{\alpha}{2}} \sin \frac{\alpha}{2} \pi\right)\right. \\
& \left.\times\left(m^{2}+r\right)^{\frac{1}{2}\left(\frac{d}{2}-1\right)} K_{\frac{d}{2}-1}\left(\left(m^{2}+r\right)^{\frac{1}{2}}|x|\right)\right] \\
= & \frac{1}{\pi(2 \pi)^{\frac{d}{2}}|x|^{\frac{d-1}{2}}} \int_{0}^{\infty} d r\left[\left(m^{\alpha}-r^{\frac{\alpha}{2}} \cos \frac{\alpha}{2} \pi\right) \sin \left(t r^{\frac{\alpha}{2}} \sin \frac{\alpha}{2} \pi\right)+r^{\frac{\alpha}{2}} \sin \frac{\alpha}{2} \pi \cos \left(t r^{\frac{\alpha}{2}} \sin \frac{\alpha}{2} \pi\right)\right] \\
& \times e^{t\left(m^{\alpha}-r^{\frac{\alpha}{2}} \cos \frac{\alpha}{2} \pi\right)}\left(m^{2}+r\right)^{\frac{1}{2}\left(\frac{d}{2}-1\right)} K_{\frac{d}{2}-1}\left(\left(m^{2}+r\right)^{\frac{1}{2}}|x|\right) .
\end{aligned}
$$

Then by the fact (2.7), we have, as $t \downarrow 0$,

$$
\begin{aligned}
n^{m, \alpha}(t, x)=\frac{1}{t} k_{0}^{m, \alpha}(t, x) & \\
& \left.\rightarrow \frac{d}{d t} k_{0}^{m, \alpha}(t, x)\right|_{t=0}=: n^{m, \alpha}(x) \\
& =\frac{\sin \frac{\alpha}{2} \pi}{\pi(2 \pi)^{\frac{d}{2}}|x|^{\frac{d}{2}-1}} \int_{0}^{\infty} d r\left(m^{2}+r\right)^{\frac{1}{2}\left(\frac{d}{2}-1\right)} r^{\frac{\alpha}{2}} K_{\frac{d}{2}-1}\left(\left(m^{2}+r\right)^{\frac{1}{2}}|x|\right) .
\end{aligned}
$$


Here the integral on the last member above is equal to

$$
\begin{aligned}
& \int_{0}^{\infty}\left(m^{2}+\tau^{2}\right)^{\frac{1}{2}\left(\frac{d}{2}-1\right)} \tau^{\alpha} K_{\frac{d}{2}-1}\left(\left(m^{2}+\tau^{2}\right)^{\frac{1}{2}}|x|\right) 2 \tau d \tau \quad\left(r:=\tau^{2}\right) \\
= & 2 \int_{m}^{\infty} a^{\frac{d}{2}-1}\left(a^{2}-m^{2}\right)^{\frac{1+\alpha}{2}} K_{\frac{d}{2}-1}(a|x|) \frac{a}{\left(a^{2}-m^{2}\right)^{\frac{1}{2}}} d a \quad\left(a:=\left(m^{2}+\tau^{2}\right)^{\frac{1}{2}}\right. \\
= & \frac{2}{|x|^{\frac{1}{2}}} \int_{m}^{\infty} a^{\frac{d-1}{2}}\left(a^{2}-m^{2}\right)^{\frac{\alpha}{2}} K_{\frac{d}{2}-1}(a|x|)(a|x|)^{\frac{1}{2}} d a .
\end{aligned}
$$

Then we use the following formula [EMOT54, Chap.X (K-Transforms), 10.2.(13), p.129]:

$$
\begin{gathered}
\int_{a}^{\infty} x^{\frac{1}{2}-\nu}\left(x^{2}-a^{2}\right)^{\mu} K_{\nu}(x y)(x y)^{\frac{1}{2}} d x=2^{\mu} a^{\mu-\nu+1} y^{-\mu-\frac{1}{2}} \Gamma(\mu+1) K_{\mu-\nu+1}(a y), \\
y>0, \mu>-1,
\end{gathered}
$$

reading with $\mu=\frac{\alpha}{2},-\nu=\frac{d}{2}-1$ and with " $\nu$ " in place of " $-\nu$ " because $K_{-\nu}(\tau)=K_{\nu}(\tau)$. to finally obtain

$$
\begin{aligned}
n^{m, \alpha}(x) & =\frac{\sin \frac{\alpha}{2} \pi}{\pi(2 \pi)^{\frac{d}{2}}|x|^{\frac{d}{2}-1}} 2^{\frac{\alpha}{2}+1} m^{\frac{d+\alpha}{2}}|x|^{-\left(\frac{\alpha}{2}+1\right)} \Gamma\left(\frac{\alpha}{2}+1\right) K_{\frac{d+\alpha}{2}}(m|x|) \\
& =\frac{2^{1+\frac{\alpha}{2}} \sin \left(\frac{\alpha}{2} \pi\right)(2 \pi)^{\frac{\alpha}{2}} \Gamma\left(\frac{\alpha}{2}+1\right)}{\pi}\left(\frac{m}{2 \pi}\right)^{\frac{d+\alpha}{2}} \frac{K_{\frac{d+\alpha}{2}}(m|x|)}{|x|^{\frac{d+\alpha}{2}}} .
\end{aligned}
$$

If $\alpha=1$, this expression reduces to

$$
n^{m, 1}(x)=2\left(\frac{m}{2 \pi}\right)^{\frac{d+1}{2}} \frac{K_{\frac{d+1}{2}}(m|x|)}{|x|^{\frac{d+1}{2}}},
$$

which is nothing but the first formula of (2.9), and we see that $n^{m, \alpha}(x)$ tends to $n^{m, 1}(x)$, as $\alpha \uparrow 1$ since $\Gamma\left(\frac{1}{2}+1\right)=\frac{\pi^{\frac{1}{2}}}{2}$.

\section{Acknowledgments}

The work by FH is supported in part by JSPS Grant-in-Aid for Challenging Exploratory Research 15K13445, and the work by TI supported in part by JSPS Grantin-Aid for Scientific Research 23540191 and 25400166.

The authors would like to thank an anonymous referee for very careful reading of the manuscript and valuable comments improving the paper.

\section{References}

[Ap04/09] A. Applebaum: Lévy processes and Stochastic Calculus, Cambridge University Press, 2004, 2nd ed. 2009.

[BE11] A.A. Balinsky and W.D. Evans: Spectral analysis of relativistic operators, Imperial College Press, London 2011. 
[ByMaRy09] T. Byczkowski, J. Malecki and M. Ryznar: Bessel potentials, hitting distributions and Green functions, Trans. Amer.Math. Soc. 361, 4871-4900 (2009).

[CFKiSi87] H.L. Cycon, R.G. Froese, W. Kirsch and B. Simon: Schrödinger Operators with Application to Quantum Mechanics and Global Geometry, Springer, BerlinHeidelberg-New York 1987.

[DeRiSe91] G.F. DeAngelis, A. Rinaldi and M. Serva: Imaginary-time path integral for a relativistic spin-(1/2) particle in a magnetic field, Europhys. Lett. 14, 95-100 (1991).

[DeSe90] G.F. DeAngelis and M. Serva: On the relativistic Feynman-Kac-Ito formula, J. Phys. A: Math. Gen. 23, L965-L968 (1990).

[EMOT54] A. Erdelyi, W. Magnus,F. Oberhettinger and F.G. Tricomi: Tables of Integral Transforms, Vol. II, Based, in part, on notes left by Harry Bateman, McGrawHill, New York-Toronto-London, 1954.

[FLSei08] R.L. Frank, E.H. Lieb and R. Seiringer: Hardy-Lieb-Thirring inequalities for fractional Schrödinger operators, J. Amer. Math. Soc. 21, 925-950 (2008).

[G10] L. Grafakos: Classical Fourier analysis, 2nd ed. Springer, New York, 2010.

[GrR94] I.S. Gradshteyn and I.M. Ryzhik: Table of Integrals, Series, and Products. translated from the fourth Russian edition, fifth ed. Translation edited and with a preface by Alan Jeffrey, Academic Press, MA, 1994

[He77] I.W. Herbst: Spectral theory of the operator $\left(p^{2}+m^{2}\right)^{1 / 2}-Z e^{2} / r$, Commun. Math. Phys. 53 (1977), no. 3, 285-294.

[HILo12] F. Hiroshima, T. Ichinose and J. Lörinczi: Path integral representation for Schrödinger operators with Bernstein functions of the Laplacian, Rev. Math. Phys. 24, 1250013(40 pages) (2012).

[HILo13] F. Hiroshima, T. Ichinose and J. Lörinczi: Probabilistic representation and fall-off of bound states of relativistic Schrödinger operators with spin 1/2, Publ. RIMS Kyoto University 49, 189-214 (2013).

[I89] T. Ichinose: Essential selfadjointness of the Weyl quantized relativistic Hamiltonian, Ann. Inst. Henri Poincaré, Phys. Théor., 51, 265-298(1989).

[I93] T. Ichinose: Note on the kinetic energy inequality leading to Lieb's negative ionization upper bound, Lett. Math. Phys. 28, 219-230(1993).

[I12] T. Ichinose: On three magnetic relativistic Schrödinger operators and imaginarytime path integrals, Lett. Math. Phys. 101, 323-339 (2012).

[I13] T. Ichinose: Magnetic relativistic Schrödinger operators and imaginary-time path integrals, Mathematical Physics, Spectral Theory and Stochastic Analysis, Operator Theory: Advances and Applications 232, pp. 247-297, Springer/Birkhäuser 2013.

[ITa86] T. Ichinose and H. Tamura: Imaginary-time path integral for a relativistic spinless particle in an electromagnetic field, Commun. Math. Phys. 105, 239-257 (1986).

[ITs92] T. Ichinose and T. Tsuchida: On Kato's inequality for the Weyl quantized relativistic Hamiltonian, Manuscripta Math. 76, 269-280 (1992). 
[IfMP07] V. Iftimie, M. Măntoiu and R. Purice: Magnetic pseudodifferential operators, Publ. RIMS Kyoto Univ. 43, 585-623 (2007).

[IkW62] N. Ikeda and S. Watanabe: On some relations between the harmonic measure and the Lévy measure for a certain class of Markov processes, J. Math. Kyoto Univ. 2 (1962), 79/95.

[IkW81/89] N. Ikeda and S. Watanabe: Stochastic Differential Equations and Diffusion Processes, North-Holland Mathematical Library, 24, North-Holland Publishing Co., Amsterdam; Kodansha, Ltd., Tokyo, 1981, 2nd ed. 1989.

[K72] T. Kato: Schrödinger operators with singular potentials, Proceedings of the International Symposium on Partial Differential Equations and the Geometry of Normed Linear Spaces (Jerusalem, 1972), Israel J. Math. 13, 135-148 (1973).

[K76] T. Kato: Perturbation Theory for Linear Operators, 2nd ed., Springer, BerlinHeidelberg-New York 1976.

[LLos01] E.H. Lieb and M. Loss: Analysis, Graduate Studies in Mathematics, 14, 2nd ed., American Mathematical Society, Providence, RI,2001.

[LSei10] E.H. Lieb and R. Seiringer: The Stability of Matter in Quantum Mechanics, Cambridge University Press, Cambridge 2010.

[LoHBe11] J. Lőrinczi, F. Hiroshima and V. Betz: Feynman-Kac-type Theorems and Gibbs Measures on Path Space, Walter de Gruyter, Berlin/Boston 2011.

[MSc13] C. Muscalu and W. Schlag: Classical and Multilinear Harmonic Analysis, II, Cambridge Studies Adv. Math. 137, Cambridge University Press, Cambridge 2013.

[N00] M. Nagasawa: Stochastic Processes in Quantum Physics, Monographs in Math. Vol. 94, Birkhäuser, Basel 2000.

[NaU90] M. Nagase and T. Umeda, Weyl quantized Hamiltonians of relativistic spinless particles in magnetic fields, J. Functional Analysis 92, 136-154(1990).

[ReSi75] M. Reed and B. Simon: Methods of modern mathematical physics II, Fourier analysis, self-adjointness, Academic Press, New York-London 1975.

[ReSi78] M. Reed and B. Simon: Methods of modern mathematical physics IV, Analysis of Operators, Academic Press, New York-London 1978.

[Sa99] K.-I. Sato: Lévy Processes and Infinite Divisibility, Cambridge University Press 1999.

[Si77] B. Simon: An abstract Kato's inequality for generators of positivity preserving semigroups, Indiana Univ. Math. J. 26, 1067-1073 (1977).

[Si79] B. Simon: Maximal and minimal Schrödinger forms, J. Operator Theory 1 (1979), 37-47.

[Si82] B. Simon: Schrödinger semigroups, Bull. (New Series) Amer. Math. Soc. 7 (1982), 447-526.

[Si79/05] B. Simon: Functional Integration and Quantum Physics, 1979, 2nd ed., AMS Chelsea Publishing, Providence, RI, 2005.

[St70] E. M. Stein, Singular Integrals and Differntiability Properties of Functions, Princeton University Press, 1970. 
[W74] R.A. Weder: Spectral properties of one-body relativistic spin-zero Hamiltonians, Ann. Inst. H. Poincaré, Sect. A (N.S.) 20 (1974), 211-220.

[Y78] K. Yosida: Functional Analysis, fifth edition, Springer, Berlin-HeidelbergNewYork 1978. 